Research Paper

\title{
Centromere Protein F (CENPF) Serves as a Potential Prognostic Biomarker and Target for Human Hepatocellular Carcinoma
}

\author{
Yugang Huang1, Xiuwen Chen ${ }^{1}$, Li Wang ${ }^{1}$, Tieyan Wang ${ }^{1}$, Xianbin Tang ${ }^{\circledR}$, and Xiaomin Su ${ }^{2^{\bowtie}}$ \\ 1. Department of Pathology, Taihe Hospital, Hubei University of Medicine, Hubei 44200, China. \\ 2. Department of Immunology, Nankai University School of Medicine, Tianjin 300110, China. \\ $\triangle$ Corresponding author: Xiaomin $\mathrm{Su}, \mathrm{PhD}$, Department of Immunology, Nankai University School of Medicine, Tianjin 300110, China. Email: \\ xiaominsu@nankai.edu.cn; and Xianbin Tang, MD, Department of Pathology, Taihe Hospital, Hubei University of Medicine, Hubei 442000, China; Email: \\ 13540409443@163.com
}

(0) The author(s). This is an open access article distributed under the terms of the Creative Commons Attribution License (https://creativecommons.org/licenses/by/4.0/). See http://ivyspring.com/terms for full terms and conditions.

Received: 2020.08.19; Accepted: 2021.03.03; Published: 2021.03.15

\begin{abstract}
Overexpression of Centromere Protein F (CENPF) is associated with tumorigenesis of many human malignant tumors. But the molecular mechanism and prognostic value of CENPF in patients with hepatocellular carcinoma (HCC) are still unclear. In this essay, expression of CENPF in HCC tumors were evaluated in a series of databases, including GEO, TCGA, Oncomine, GEPIA, The Human Protein Atlas and Kaplan-Meier plotter. It was apparent that mRNA and protein expression levels of CENPF were significantly increased in patients with $\mathrm{HCC}$ and were manifestly associated with the tumor stage of $\mathrm{HCC}$. Aberrant expressions of CENPF were significantly linked with worse overall survival (OS) and progression-free survival (PFS) in HCC patients. Then, immunohistochemistry of CENPF in human HCC samples was carried out to suggest that CENPF protein was over-expressed in HCC tissues, compared with paired adjacent non-cancerous samples. And small interfering RNAs of CENPF in the human HepG2 cells were further performed to reveal that down-regulation of CENPF significantly inhibited cell proliferation, cell migration, and cell invasion, but slightly promoted cell apoptosis in human HepG2 cells. Moreover, the gene-set enrichment analysis (GSEA) was conducted to probe the biology process and molecular signaling pathway of CENPF in HCC. The GSEA analysis pointed out that CENPF was principally enriched in cell cycle and closely related to $E 2 F I$ and $C D K I$ in the regulation of cell cycle, especially during G2/M transition of mitosis in HCC. Additionally, immune infiltration analysis by CIBERSORTx revealed that mutilpe immune cells, including $\mathrm{T}_{\text {reg, }}$ etc., were significantly different in $\mathrm{HCC}$ samples with CENPFhigh, compared with CENPFlow. These results collectively demonstrated that CENPF might serve as a potential prognostic biomarker and novel therapeutic target for HCC. However, further research is needed to validate our findings and promote the clinical application of CENPF in HCC.
\end{abstract}

Key words: CENPF, HCC, hepatocellular carcinoma, biomarker, survival, prognostic value, bioinformatics analysis.

\section{Introduction}

Hepatocellular carcinoma (HCC), as the most common primary liver neoplasms, is one of the most malignant tumors with high morbidity and mortality, which makes it a notable healthcare issue for human beings in the global world [1,2]. Liver neoplasms are the fourth leading cause of cancer-related death and ranks sixth among new cases worldwide [3, 4]. Surpassing breast, prostate, and colorectal cancers, liver neoplasms is predicted to be the third leading cause of cancer-related death in Europe and the United States by 2030 [5, 6]. The World Health Organization estimates that the global incidence of $\mathrm{HCC}$ is rising and might reach one million cases annually in the next decade [7, 8]. HCC has high molecular heterogeneity, with a poor prognosis [9, 10]. Due to the lack of effective biomarkers to detect 
diseases and predict individual differences in patients, the mortality rate of HCC is high. Over $80 \%$ of patients are diagnosed with advanced liver cancer $[11,12]$. As the 5 -year survival rate is only $18 \%$, liver cancer is the second most fatal tumor after pancreatic cancer [13-15]. For patients in Asian countries such as China, the situation is even more severe, with a 5-year survival rate reported as low as $12 \%[16,17]$. So far, some biomarkers with potential diagnostic, prognostic or therapeutic value for HCC have been reported. Molecular studies [18-20] have shown that the most common variations in HCC include mutations in the TERT promoter, TP53 and CTNNB1, copy number variation, abnormal DNA methylation [21], overexpression of PD-L1 [22-24], etc [25]. Although some of the above-mentioned biomarkers of HCC have aroused extensive concern, most of them were studied separately rather than as a part of the whole carcinogenesis process; the related studies are still in the preliminary investigation or clinical verification stage. Therefore, it is urgent to find reliable biomarkers to predict the early or accurate prognosis, and to develop new molecular targeted therapy strategies for HCC.

As a cell cycle-related nuclear antigen, the Centromeric protein $\mathrm{F}$ (CENPF) is expressed at low levels in G0/G1 cells and accumulates in the nuclear matrix in the S-phase, with the highest expression level in G2/M cells [26]. The abnormal expression or activation of CENPF has been reported in several human malignant tumors, including $\operatorname{HCC}[27,28]$, breast cancer [29], and other tumors [30]. Additionally, elevated CENPF expression contributes to unregulated cell proliferation in HCC [28]. Recent studies have shown that CENPF and FOXM1 were important regulators of prostate cancer malignancies and prognostic indicators for poor survival and extensive tumor metastasis [31]. Further studies have shown that COUP transcription factor 2 promoted prostate cancer metastasis through CENPF signal transduction [32]. Generally, CENPF might emerge as a promising biomarker for predicting the prognosis of HCC. Therefore, identifying the masked mechanism of CENPF-mediated oncogenes or tumor suppressor genes as predictive biomarkers might provide new treatment strategies. Nevertheless, the divergences in expression levels, genetic alterations, biological functions and process, molecular mechanisms, and prognostic value of CENPF in HCC have not been fully expounded.

The advancement and development of gene microarray and RNA-sequencing technology has innovated the research of RNA and DNA, which has become an important method for biological and medical research [33-35]. Based on the GEO,
Oncomine and TCGA databases, this study expanded the relevant knowledge of HCC and comprehensively analyzed the relationship between CENPF and the pathogenesis and progression of HCC, in order to provide useful enlightenment for the occurrence and aggressiveness of HCC.

\section{Materials and Methods}

\section{Data resource and description}

As a publicly available genomics database, Gene Expression Omnibus (GEO) of NCBI was systematic and complete queried for all datasets related to studies of HCC, which collects submitted high-throughput gene expression data worldwide. Following criteria were considered qualified for our analysis: (1) The research objects included human HCC and its adjacent or normal liver tissue. (2) The information of technology and platform used for studies was detailed. (3) The number of samples was $>10$. Based on these criteria, eight gene expression microarray datasets for HCC, including GSE14520, GSE60502, GSE40367, GSE84005, GSE112791, GSE76297, GSE25097, and GSE87630, were downloaded from the repository.

HCC mRNA normalized counts data of TCGA, derived from RNA-seq Htseq platform, were downloaded from Genomic Data Commons (GDC) Data Portal. TCGA RNA-seq data contains 424 samples, including 374 primary HCC tumor and 50 normal liver samples. RTCGA Toolbox [36] and edgeR [37] packages were applied to detect the expression of CENPF in HCC and normal tissues.

\section{Identification CENPF expression by Bioinformatics strategy}

As the currently world's largest oncogene chip database and integrated data mining platform, Oncomine is widely used to excavate cancer gene information. So far, the database has included gene expression data from 715 datasets and about 90,000 cancer and normal tissue samples, which can be used to explore the expression of CENPF in HCC and its related normal tissues. [38]. Studies of CENPF expression in HCC and normal liver samples were selected with the thresholds as follows: $P$-value $<0.05$ and the data type was restricted to mRNA expression levels in Oncomine database and visualized by GraphPad Prism. Moreover, protein expression of CENPF in HCC tissues and their associated normal tissues was screened in The Human Protein Atlas, which is a human proteomics online service database and designed to map human proteomics information in cells, tissues, and organs by using various omics technologies, including antibody-based imaging, 
mass spectrometry-based proteomics, transcriptomics, and systems biology.

Gene Expression Profiling Interactive Analysis (GEPIA, http://gepia.cancer-pku.cn/) is an interactive online database for detecting the mRNA expression of 9736 tumors and 8587 normal samples from the TCGA and Genotype-tissue Expression dataset (GTEx) projects [39]. GEPIA can be performed for gene differential expression analysis, profiling plotting according to cancer types or pathological stages, correlation analysis, patient survival analysis, and similar gene analysis. The mRNA expression of CENPF in different stages or grades was compared between HCC and normal tissues by using the GEPIA dataset and TCGA RNA-seq data.

\section{Correlation between CENPF and clinicopathological characteristics in HCC}

Based on HCC information from TCGA datasets, correlation between CENPF and clinic-pathological characteristics was investigated. The expression matrix from TCGA datasets contains 374 HCC tissues divided to two groups, including187 HCC samples with CENPF low expression (CENPFlow) and 187 HCC samples with CENPF high expression (CENPFhigh) based on the median expression level of CENPF (median cutoffs). Grouping of risk types was conducted via 'ggrisk' package of $\mathrm{R}$ software (version $4.0 .3)[40,41]$. Sanguini diagram, which can be used to show the distribution trend of survival and expression of a gene in different stages, ages and other clinical characteristics for tumors, was built based on the 'ggalluval' package of R software [42].

Further, the univariate (uni-cox) and multivariate cox (mult-cox) regression analysis was performed to identify the proper terms to build the nomogram. The forest was used to show the P-values, $\mathrm{HR}$ and $95 \%$ confidence interval $(\mathrm{CI})$ of each variable through 'forestplot' $\mathrm{R}$ package. A nomogram was developed based on the results of multivariate Cox proportional hazards analysis to predict the 1-year, 2-year, and 3-year overall recurrence [43]. The nomogram provided a graphical representation of the factors, which can be used to calculate the risk of recurrence for an individual patient by the points associated with each risk factor through ' $r m s^{\prime} \mathrm{R}$ package [44].

\section{Survival analysis}

Kaplan-Meier plotter is an online data service platform that contains microarray gene expression data and survival information from GEO, TCGA, and the Cancer Biomedical informatics Grid, which provides survival information of 374 HCC patients [45]. In this study, the Kaplan-Meier plotter was conducted to evaluate the prognostic value of CENPF mRNA expression. The overall survival (OS), progression-free survival (PFS), 1-year, 3-year and 5 -year OS of HCC patients were tested by dividing samples into two groups based on median expression (high expression and low expression) and assessed by using Kaplan-Meier survival plots, with a hazard ratio with 95\% confidence intervals and log rank $P$-value. Further, subgroup survival analyses were conducted by dividing patients based on different population, pathological and histological subtypes.

\section{Gene-set enrichment analysis (GSEA) in HCC samples}

In order to interpret the gene expression data between CENPF and other genes, and to identify the underlying pathways that correlate to HCC with CENPFlow or CENPFhigh, GSEA software (version 4.0.3, Broad Institute, USA) was performed, to probe the biological mechanisms based on TCGA datasets $[46,47]$. The predefined gene sets, including 'c2.cp.kegg.v7.2.symbols.gmt', 'c2.cp.biocarta.v7.2. symbols.gmt', and 'h.all. v7.2.symbols.gmt', from the Molecular Signatures Database were employed, respectively. A normalized enrichment score (NES) was calculated as the primary GSEA statistic. For the analysis results, the threshold values of statistical significance were set as $|\mathrm{NES}|>1$, normalized P-values (NOM P-values) $<0.05$, and FDR $<0.25$. Lastly, the results of the GSEA analysis were visualized via Sangerbox tools, a free online platform for data analysis (http:/ / www.sangerbox.com/tool).

\section{Analysis of immune cell infiltration profile}

Immune cell infiltration is an important index to predict immunotherapy [48]. Based on TCGA dataset, analysis of immune cell infiltration was performed by CIBERSORTx, an analytical tool that provides an estimation of the abundances of member cell types in a mixed cell population by inputting normalized gene expression matrix [49]. The results of the analysis present the abundance of 22 kinds of immune cells, including 7 types of T cells, 3 types of B cells, 2 types of natural killer (NK) cells, monocytes, 3 types of macrophages $(\mathrm{M} \varphi), 2$ types of dendritic cells (DC), 4 types of granulocytes (mast cells, eosinophils, and neutrophils). The expression matrix from TCGA dataset was normalized via 'Limma' package of $\mathrm{R}$ software, and all the results were visualized by applying the packages of $\mathrm{R}$ software. Moreover, the expression of five genes closely related to tumor immunotherapy, including LAG3, CTLA4, HAVCR2, $P D-1$, and $P D-L 1$ were further investigated in HCC by GEPIA [50]. 


\section{Analysis of the correlation between CENPF expression and MSI or TMB in HCC}

As independent factors of immune response to PD-1 / PD-L1 mono-antibody, the microsatellite instability (MSI) and tumor mutation burden (TMB) have been clinically proven to play an outstanding role in predicting the anti-tumor effect of PD-1 / PD-L1 inhibitors [51, 52]. The analysis of the correlation between CENPF expression and TMB/MSI in HCC based on TCGA were performed via 'ggstatsplot' package of $\mathrm{R}$ software. Spearman's correlation analysis was used to describe the correlation between quantitative variables without a normal distribution. $P$-value $<0.05$ was considered statistically significant. The range of correlation coefficient is $(-1,1)$. A negative number represents a negative correlation between the two gene expressions, and a positive value represents a positive correlation. The closer the value is to 1 or -1 , the stronger the correlation between the two variables; the closer the value is to 0 , the weaker the correlation between the two variables.

\section{Human HCC samples and immunohisto- chemistry}

Screening criteria for PPFE (Formalin-fixed and paraffin embedded) samples of HCC patients were as follows: (1) All samples were stored at room temperature $\left(20 \sim 25^{\circ} \mathrm{C}\right)$ and collected from patients who had been diagnosed as HCC from May 2019 to May 2020 at Taihe Hospital of Hubei University of Medicine, China. All HCC patients were diagnosed and graded according to the pathological characteristics by at least two pathologists in the Department of Pathology, Taihe Hospital, with a total of 81 cases. (2) Samples containing cancerous tissues and paired adjacent non-cancerous samples were screened out. Finally, 5 HCC tissues and 5 paired adjacent non-cancerous samples of PPFE were included. All human samples were obtained by informed consent (IFC) from patients or family members, and this study was supported and approved by the Ethics Committee of Taihe Hospital. Details of 5 enrolled patients with HCC were listed in Table S1.

Immunohistochemistry of paraffin embedded samples (IHC-P) was performed according to the manufacturer's recommended procedure. $3 \mu \mathrm{m}$ of HCC tissue and para-cancerous tissue were taken from the PPFE. All sections were deparaffinized with xylene and rehydrated through a graded ethanol series. Endogenous peroxidase activity was blocked with 3\% hydrogen peroxide in methanol for $10 \mathrm{~min}$. Antigen retrieval was performed by EDTA at $\mathrm{pH} 9.0$ in a pressure cooker for $4 \mathrm{~min}$. After PBS washing (three times, $3 \mathrm{~min}$ each), slides were incubated with the rabbit anti-human CENPF polyclonal antibody (ab5, Abcam, 1/100 dilution) at $37^{\circ} \mathrm{C}$ for 1 hour. After incubation with HRP labeled second antibody at $37^{\circ} \mathrm{C}$ for 0.5 hour, nuclei were stained by hematoxylin for 30 seconds.

\section{Cell line and siRNA}

Small interfering RNAs of CENPF (siCENPF) was designed to explore the role of CENPF in cell proliferation and migration of HCC in the hepatoma cell line, human HepG2. The qRT-PCR primers and small interfering RNAs of CENPF and reference gene were synthesized by Sangon Biotech (Shanghai, China), and the information of their sequences was listed in Table 1.

The small interfering RNA transfection was performed according to instructions of the manufacturer. Each group had at least 3 replicates.

\section{RNA isolation and quantitative real-time PCR}

HepG2 cells after cells were transfected with 50 nmol of CENPF siRNA (siCENPF) or contorl siRNA (siNC) for 48 hours respectively. Total RNA was extracted from the HepG2 cells using TRIzol reagent (Invitrogen, USA). First-strand cDNA was generated from total RNA using oligo-dT primers and reverse transcriptase (Invitrogen, USA). Quantitative real-time PCR (qRT-PCR) was conducted using QuantiTect SYBR Green PCR Master Mix (Qiagen, Germany) and specific primers in an ABI Prism 7000 analyzer (Applied Biosystems, USA). GAPDH was detected in each experimental sample as an endogenous control. All the reactions were run in triplicate. The relative RNA levels of CENPF in HCC samples were calculated by using the $2^{-\Delta \Delta \mathrm{Ct}}$ method.

\section{Western blotting}

For western blotting, human HepG2 cells were transfected with $50 \mathrm{nmol}$ of siCENPF) and siNC for 48 hours respectively, and then cells were collected. Cell lysates were denatured and subjected to SDS-PAGE, then were transferred to PVDF membranes (Millipore, USA). The membranes were incubated with primary antibody (CENPF antibody: ab5, Abcam, UK) overnight at $4^{\circ} \mathrm{C}$. Membranes were washed for 4 times with washing buffer (three times TBST and at last one time TBS, 10 minutes each), and then incubated with the secondary HRP-conjugated antibody for 1.5 hour at room temperature. After washed by washing buffer for 4 times, the membranes were detected using an enhanced chemiluminescence assay with Lumi-Glo reagents (Millipore, USA). 
Table 1. The sequences information of qRT-PCR primers and siRNAs.

\begin{tabular}{lll}
\hline Gene name & Primers $\left(\mathbf{5}^{\prime} \mathbf{- 3} \mathbf{\prime}\right)$ & \\
\hline CENPF & Forward: AGCACTGATCACCTGTTAGC & Reverse: ACCCACATACAAACAGAGATTG \\
GAPDH & Forward: CGGAGTCAACGGATTTGGTCGTAT & Reverse: AGCCTTCTCCATGGTGGTGAAGAC \\
siCENPF & Forward: GACCCAGAAACUAGCUUAUTT & Reverse: AUAAGCUAGUUUCUGGGUCTT \\
siNC & Forward: UUCUCCGAACGUGUCACGUTT & Reverse: ACGUGACACGUUCGGAGAATT \\
\hline
\end{tabular}

siCENPF: CENPF siRNA, siNC: negative control siRNA.

Table 2. Details of HCC studies and associated microarray datasets from GEO database.

\begin{tabular}{|c|c|c|c|c|c|}
\hline \multirow{2}{*}{$\begin{array}{l}\text { GEO } \\
\text { Series }\end{array}$} & \multirow[t]{2}{*}{ Contributor(s) } & \multicolumn{2}{|l|}{ Sample } & \multirow[t]{2}{*}{ Platform } & \multirow[t]{2}{*}{ Country } \\
\hline & & Tumor & Normal & & \\
\hline GSE14520 & Roessler S et al, 2009 & 225 & 220 & GPL3921 [HT_HG-U133A] Affymetrix HT Human Genome U133A Array & USA \\
\hline GSE60502 & Kao KJ, 2014 & 18 & 18 & GPL96 [HG-U133A] Affymetrix Human Genome U133A Array & China (Taiwan) \\
\hline GSE40367 & Roessler S, 2012 & 10 & 5 & GPL570 [HG-U133_Plus_2] Affymetrix Human Genome U133 Plus 2.0 Array & USA \\
\hline GSE84005 & Tu X et al, 2016 & 38 & 38 & GPL5175 [HuEx-1_0-st] Affymetrix Human Exon 1.0 ST Array & China \\
\hline GSE112791 & Kaoru Mogushi et al, 2018 & 183 & 15 & GPL570 [HG-U133_Plus_2] Affymetrix Human Genome U133 Plus 2.0 Array & Japan \\
\hline GSE76297 & Xin Wei W, 2015 & 61 & 151 & GPL17586 [HTA-2_0] Affymetrix Human Transcriptome Array 2.0 & USA \\
\hline GSE25097 & Zhang C, 2010 & 268 & 243 & GPL10687 Rosetta/Merck Human RSTA Affymetrix 1.0 microarray, Custom CDF & USA \\
\hline GSE87630 & Woo HG, 2016 & 64 & 30 & GPL6947 Illumina HumanHT-12 V3.0 expression & South Korea \\
\hline
\end{tabular}

\section{CCK-8 cell proliferation experiment}

Human HepG2 cells were plated in 96-well plates at $100 \mu \mathrm{L}$ (total $2 \times 10^{3}$ cells), then transfected with $50 \mathrm{nmol}$ of siCENPF and siNC respectively. 10 $\mu \mathrm{L}$ CCK-8 solution (Beyotime, China) was added to each well. The cells were incubated in the cell incubator for 0.5 hour and 1 hour. The absorbance (optical density, OD) representing cell density was measured at $450 \mathrm{~nm}$.

\section{Wound healing assay}

Cell migration was analyzed in wound healing assay. Human HepG2 cells were seeded in 12-well plates in DMEM with $10 \%$ fetal bovine serum (FBS), then cells were transfected with $50 \mathrm{nmol}$ of siCENPF or siNC for 24 hours. Wounds were scratched by $20 \mu l$ pipette tips. Each well was then rinsed 5 times with PBS to clear floating cells from scratches, and $3 \mathrm{~mL}$ of $10 \%$ FBS, $1 \%$ antibiotic-antimycotic DMEM was added to each well. Scratch regions were photographed at 0 and 24 hour.

\section{Transwell assay}

Transwell assays were performed to analysis metastatic ability and invasion of the HepG2 cells. The metastatic ability of the cells was investigated by Transwell plates (Corning, USA). Cells were transfected with $50 \mathrm{nmol}$ of siRNAs for 24 hours. Then Serum-free single cell suspensions were placed in the upper chamber per well. The lower chamber was filled with $500 \mathrm{uL} 1640$ medium with $20 \%$ FBS as a chemoattractant for 24 hours. For invasion assays, the membrane inserts were pre-coated with Matrigel. The cells were cultured for $24 \mathrm{~h}$. Cells in the lower surface of the membrane was fixed with $4 \%$ PFA and then stained with $0.5 \%$ crystal violet. Cells in ten random fields per chamber were counted and analyzed using Image J software. The percentage of migration was calculated and compared to the mock group.

\section{Flow cytometric analysis}

Cells were transfected with $50 \mathrm{nmol}$ of siRNAs for 24 hours. Then cells were analyzed using the Annexin V-FITC apoptosis detection kit (Vazyme Biotech, USA) as instrument. $1 \times 10^{4}$ cells of each sample were counted in the flow cytometer (BD, Bangladesh).

\section{Statistical analysis}

Statistical analysis was performed via GraphPad Prism (version 8.2.1, San Diego, CA) and SPSS 22.0 (IBM SPSS Inc. Chicago, IL) software. Student's t-test (two-tailed) were utilized for the comparison of two sample groups. Differences were considered as statistically significant when $P<0.05\left({ }^{*} P<0.05\right.$, ${ }^{* *} P<$ 0.01 , $\left.{ }^{* * *} P<0.001, * * * * P<0.0001\right)$.

\section{Results}

\section{CENPF is overexpressed in HCC compared with normal liver tissues}

The details of GEO series involved in this study were presented in Table 2. As illustrated in Figure 1, CENPF mRNA was significantly overexpressed in GSE14520, GSE60502, GSE40367, GSE84005, GSE112791, GSE76297, GSE25097, and GSE87630 (all $P<0.01$ ). For further validation, we performed meta-analysis of CENPF expression in Oncomine database. Compared with that in normal livers, CENPF was evidently upregulated in HCC tumors $(P$ $<0.001$, Figures 2A-D), but not in cirrhosis, and liver cell dysplasia $(P>0.05$, Figure $2 \mathrm{D})$. Moreover, study from The Human Protein Atlas declared that protein 
of CENPF is mainly expressed in the region of nucleoplasm by immunofluorescence (IFC) staining in MCF7 cells (Figure 2E). And CENPF was also high expressed in HCC patients (Figure 2F) or cell line, human HepG2 cells (Figure 2G), compared to normal ones in terms of protein or mRNA. The relationship between the transcription levels of CENPF and the tumor stage/grade in HCC patients were also analyzed by the GEPIA (Figure 3A) and TCGA (Figures 3B-C) dataset. As results, the mRNA expression level of CENPF was significantly and positively correlated with the tumor stage and grade for HCC.

\section{Correlation between CENPF and clinicopathological characteristics in patients with HCC}

In order to explore the correlation between CENPF and clinicopathological characteristics in patients with HCC, all 374 HCC tissues were divided into two groups, including187 CENPFlow HCC samples and 187 CENPFhigh HCC samples according to the median cutoffs of CENPF expression (Figure 4A). As listed in Table 3, BMI of all HCC patients were over $18.5 \mathrm{~kg} / \mathrm{m}^{2}$, and more HCC cases had higher BMI in CENPF high group than those in CENPF low group $(P=0.025)$. Less HCC cases had family history of cancer in CENPF high group than those cases in CENPF low group $(27.8 \%$ vs. $37.4 \%, P=0.036)$.

Incidence of new tumor events after initial treatment was higher in CENPF high group than those in CENPF low group $(P=0.038)$. However, HCC patients in CENPF low group suffered from less hepatic inflammation ( $39.6 \%$ vs $29.4, P=0.029)$. Just as we expected, HCC patients with CENPFhigh group experienced manifestly advanced neoplasm histologic grade (especially grade III, $P<0.01$ ) and advanced pathological stage (especially stage II and III, $P=$ 0.032). Meanwhile, the distribution of CENPF expression in gender, pTNM stage and grade was shown in Figure 4B. Furthermore, the uni-cox and mult-cox regression analysis was performed to identify the effects of CENPF and clinical factors including age, gender, grade, pTNM stage, and new tumor events on the prognosis of HCC patients. According to the uni-cox analysis in Figure 4C, the CENPF expression and pTNM stage were correlated with the prognosis of HCC patients (all $P<0.05)$. And according to the mult-cox analysis in Figure $4 \mathrm{D}$, the CENPF expression and pTNM stage might be independent prognostic factors in HCC patients (all $P<0.05)$. Based on uni-cox and mult-cox analysis, nomogram was constructed to predict 1-year, 2- year, and 3- year survival rate in one HCC patients associated with CENPF expression and pTNM stage (Figure 4E).

\section{GSE40367}

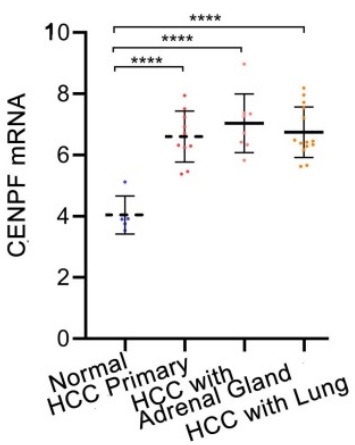

G. GSE25097

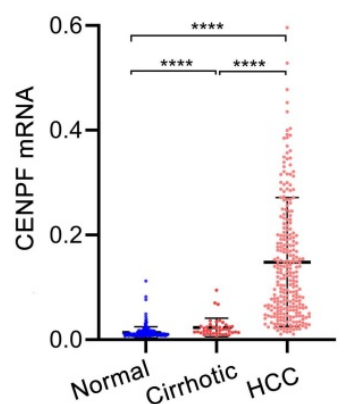

D. GSE84005

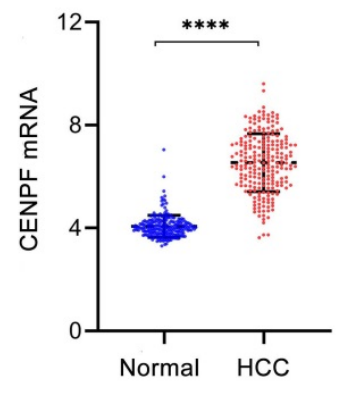

H. GSE87630

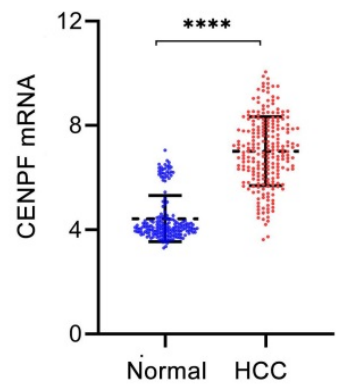

Figure 1. The expression of CENPF in HCC patients from GEO datasets. Relative mRNA expression levels of CENPF from (A) GSE14520, (B) GSE60502, (C) GSE40367, (D) GSE84005, (E) GSE1 12791, (F) GSE76297, (G) GSE25097, and (H) GSE87630 in normal livers and HCC samples. Pre-processed expression levels are Log 2 normalized and median centered. Data were analyzed using un-paired student's t-test. Differences were viewed as statistically significant when $P<0.05$. HCC: hepatocellular carcinoma. HCC with Adrenal Gland: $\mathrm{HCC}$ with adrenal gland metastasis. $\mathrm{HCC}$ with Lung: $\mathrm{HCC}$ with lung metastasis. 
A

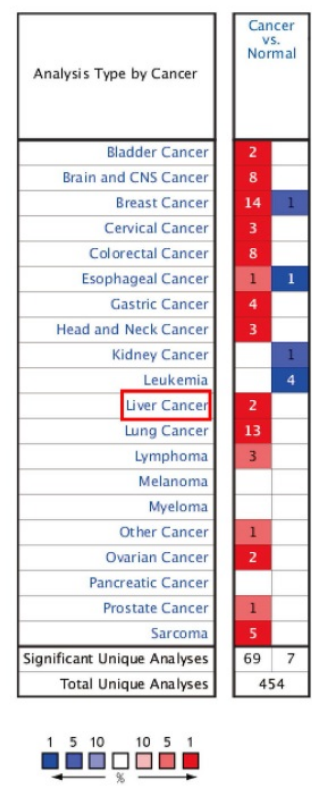

B. Meta-analysis of CENPF in 3 analyses

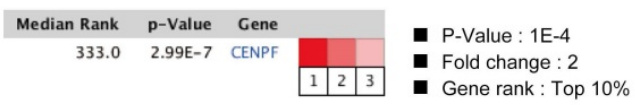

Legend

1. Hepatocellular Carcinoma vs. Normal 3. Liver Cancer Type: Hepatocellular

Chen Liver, Mol Biol Cell, $2002 \quad$ Carcinoma

2. Hepatocellular Carcinoma vs. Normal Wurmbach Liver, Hepatology, 2007

Wurmbach Liver, Hepatology, 2007

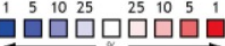

The rank for a gene is the median rank for that gene across each of

the analyses.

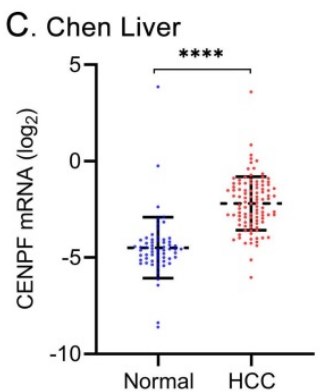

\section{Wurmbach Liver}

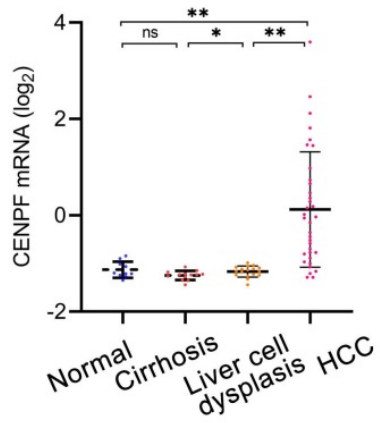

$\mathrm{F}$

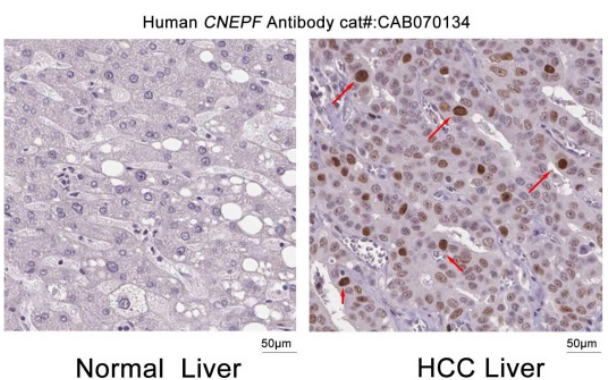

Normal Liver
G

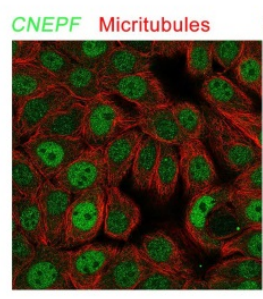

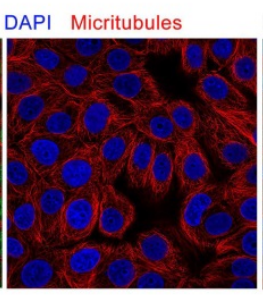

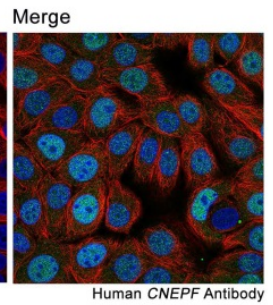

catt: HPA052382

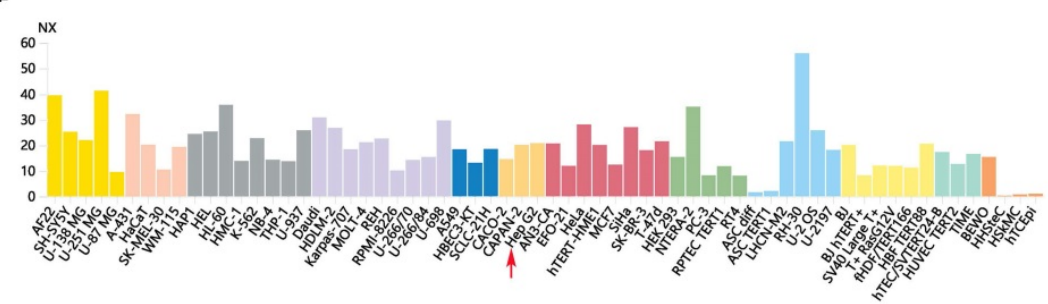

Figure 2. Comparison of CENPF expression in Oncomine and The Human Protein Atlas database. (A) The mRNA levels of CENPF in different types of cancers from Oncomine database. The graph shows the numbers of datasets with statistically significant mRNA over-expression (red) or down-regulated expression (blue) of CENPF. The threshold was designed as following parameters: $P$-value $<0.05$ and fold change $\geq 1.5$; (B) Meta-analysis of CENPF expression in 3 analyses; (C) CENPF levels in normal, and HCC tissues in Chen Liver; (D) CENPF levels in normal, cirrhosis, liver cell dysplasia and HCC tissues in Wurmbach Liver; (E) Protein expression and localization of CENPF in MCF7 cell line by IFC; IFC: immunofluorescence. Green represents the protein CENPF, red represents microtubules, and blue represents nucleus. After merging, the protein CENPF is expressed in the nuclear region. (F) Protein expression of CENPF in normal, and HCC human tissues by IHC. The expression of CENPF was shown by the red arrow. IHC: immunohistochemistry. (G) The mRNA expression profile of CENPF in different human cell line, including HepG2 (shown by the red arrow).

A

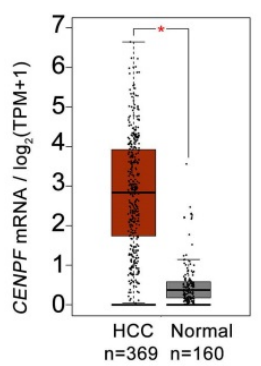

B

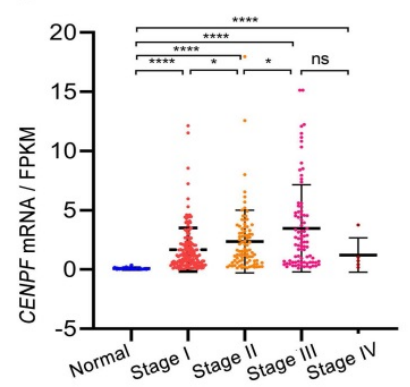

C

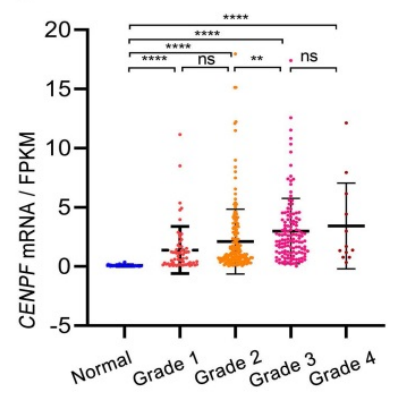

Figure 3. Correlation between the transcription levels of CENPF and the tumor stage/grade of patients with HCC. (A) The mRNA levels of CENPF in normal( $\mathrm{n}=160)$ and $\mathrm{HCC}$ tissues( $n=369$ ) from GEPIA. (B-C) The mRNA levels of CENPF in HCC in PTNM stage I-IV and grade 1-4 from TCGA database. 
A

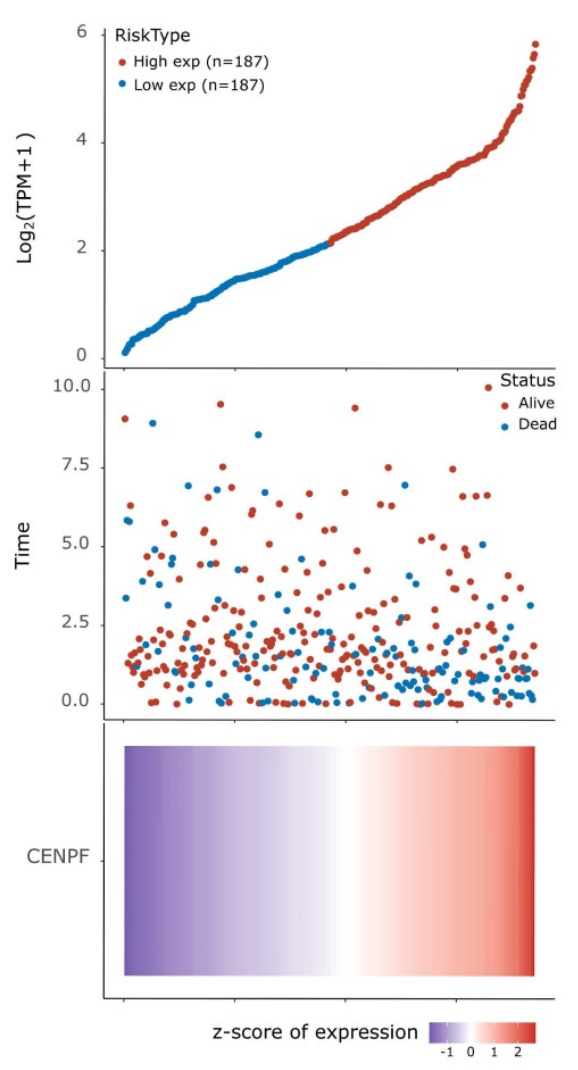

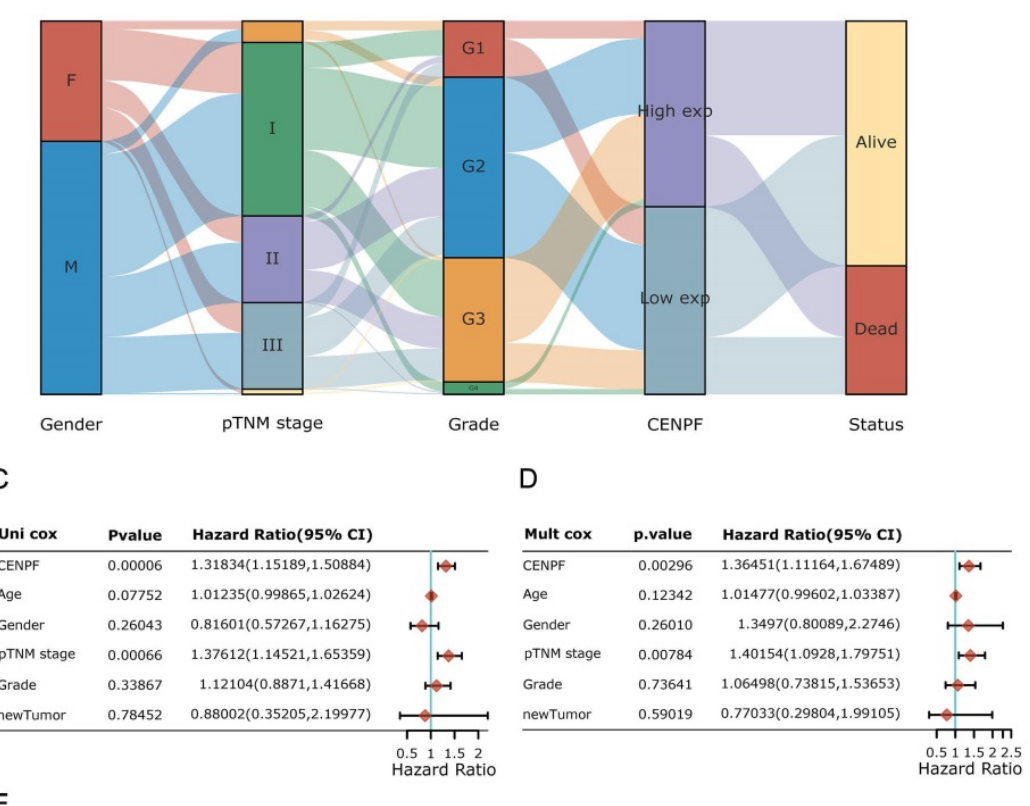

E

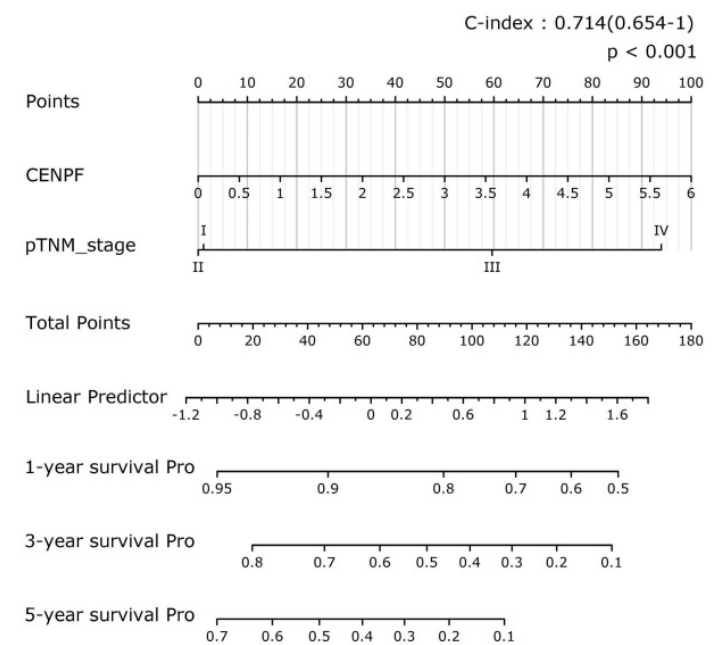

Figure 4. Correlation between the expression of CENPF and the prognosis in HCC based on TCGA datasets. (A) CENPF expression and survival status of HCC patients from TCGA datasets, in which the top represents the scatter plot of the gene expression from low to high, and is divided into two groups according to the median cutoffs; the middle represents the scatter plot distribution of survival time and survival status corresponding to gene expression of different samples; the bottom represents the heat map of the gene expression in the corresponding samples; the abscissa of the upper, middle and lower graphs in the graph represent the samples, and the sample order is consistent. (B) Sanguini diagram for outlining the distribution of CENPF expression in gender, PTNM stage and grade. Each column represents a characteristic variable, different colors represent different types or stages, and lines represent the distribution of the same sample in different characteristic variables. Hazard ratio and $P$-value of constituents involved in uni-cox (C) and mult-cox (D) regression and relevant clinical parameters of the CENPF. (E) Nomogram to predict 1-year, 2-year, and 3-year survival rate in HCC patients associated with CENPF expression and PTNM stage.

\section{Overexpression of CENPF predicts worse prognosis in HCC patients}

As validated in Kapan-Meier Plotter, overexpression of CENPF predicted worse OS (overall survival; $\mathrm{HR}=1.54, \log \operatorname{rank} P=0.013$ ) and PFS (progression-free survival; $H R=1.77, \log$ rank $P=$ 0.00013) in HCC (Figures 5A-B). Meanwhile, subgroup analyses indicated that CENPF upregulation in tumors was a risk factor for 1-year, 3 -year and 5-year OS in patients with HCC $(\mathrm{HR}=2.29$, $\log$ rank $P=0.0028 ; \mathrm{HR}=1.99, \log$ rank $P=0.00058$ and $\mathrm{HR}=1.68, \log$ rank $P=0.0044$, respectively, Figures 5C-E).
For subgroup survival analyses, upregulation of CENPF was significantly associated with poor OS in HCC patients with neoplasm histologic grade I and grade III $(\mathrm{HR}=5.84, \log \operatorname{rank} P=0.00064$ and $\mathrm{HR}=$ $2.46, \log$ rank $P=0.0041$, respectively, Figures $6 \mathrm{~A}$ and C), while no significant difference was found in HCC cases with grade II $(\mathrm{HR}=1.49, \log$ rank $P>0.05$, Figure 6B). In addition, the overexpression of CENPF was closely related to poor OS in HCC patients with stage I-II (HR $=1.83, \log$ rank $P=0.021$, Figure $6 \mathrm{D})$, stage II-III $(\mathrm{HR}=2.37, \log$ rank $P=3 \mathrm{E}-04$, Figure $6 \mathrm{E})$, and stage III-IV $(\mathrm{HR}=2.36, \log \operatorname{rank} P=0.0027$, Figure $6 \mathrm{~F})$. 
Table 3. Characteristics of HCC patients between CENPFhigh and CENPFlow groups (median cutoffs).

\begin{tabular}{|c|c|c|c|c|c|c|c|}
\hline \multirow[t]{2}{*}{ Variables } & \multicolumn{2}{|c|}{$\begin{array}{l}\text { CENPF expression } \\
\text { level }\end{array}$} & \multirow[t]{2}{*}{$\begin{array}{l}P \\
\text { value }\end{array}$} & \multirow[t]{2}{*}{ Variables } & \multicolumn{2}{|c|}{$\begin{array}{l}\text { CENPF expression } \\
\text { level }\end{array}$} & \multirow[t]{2}{*}{$\begin{array}{l}P \\
\text { value }\end{array}$} \\
\hline & $\begin{array}{l}\text { Low } \\
(\mathrm{n}=187)\end{array}$ & $\begin{array}{l}\text { High } \\
(\mathrm{n}=187)\end{array}$ & & & $\begin{array}{l}\text { Low } \\
(\mathrm{n}=187)\end{array}$ & $\begin{array}{l}\text { High } \\
(\mathrm{n}=187)\end{array}$ & \\
\hline Gender, male (\%) & $145(77.5)$ & $131(70.1)$ & 0.106 & Pathological stage, n (\%) & & & 0.032 \\
\hline Age, median (IQR), years & $64(15)$ & $59(17)$ & 0.196 & I & $107(57.2)$ & $84(44.9)$ & \\
\hline BMI, $\mathrm{kg} / \mathrm{m}^{2}, \mathrm{n}(\%)$ & & & 0.025 & II & $42(22.5)$ & $53(28.3)$ & \\
\hline$<18.5$ & 0 & 0 & & III & 35 (18.7) & $55(29.4)$ & \\
\hline $18.5 \sim 24.99$ & $36(19.3)$ & $18(9.6)$ & & IV & $4(2.1$ & $1(0.5)$ & \\
\hline $25 \sim 29.99$ & $106(56.7)$ & $109(58.2)$ & & NA & $16(8.6)$ & $13(7.0)$ & \\
\hline$>30$ & $46(24.6)$ & $58(31.0)$ & & Vascular invasion, $\mathrm{n}(\%)$ & & & 0.072 \\
\hline NA & $17(9.1)$ & $20(10.7)$ & & Macro & $7(3.7)$ & $11(5.9)$ & \\
\hline Tumor status, $\mathrm{n}(\%)$ & & & 0.059 & Micro & $54(28.9)$ & $51(27.3)$ & \\
\hline With tumor & $54(29.9)$ & $69(36.9)$ & & None & $122(65.2)$ & $106(56.7)$ & \\
\hline Tumor free & $139(74.3)$ & $118(63.1)$ & & NA & $21(11.2)$ & $38(20.3)$ & \\
\hline NA & $11(5.9)$ & $19(10.2)$ & & Child-pugh classification, $\mathrm{n}(\%)$ & & & 0.082 \\
\hline Family history of cancer, $\mathrm{n}(\%)$ & & & 0.036 & A & $129(68.9)$ & $112(59.8)$ & \\
\hline No & $100(53.5)$ & $127(67.9)$ & & B & $13(6.9)$ & $9(4.8)$ & \\
\hline Yes & $70(37.4)$ & $52(27.8)$ & & $\mathrm{C}$ & $1(0.5)$ & $0(0)$ & \\
\hline NA & $34(18.2)$ & $27(9.7)$ & & NA & $61(32.6)$ & $85(45.5)$ & \\
\hline Hepatocarcinoma risk factors*, n (\%) & & & 0.63 & New tumor event after initial treatment, $n(\%)$ & $90(48.1)$ & $112(59.8)$ & 0.038 \\
\hline Hepatitis virus infection & $57(30.9)$ & $65(34.8)$ & & Ishak fibrosis status, $\mathbf{n}(\%)$ & & & 0.084 \\
\hline Alcohol consumption & $75(40.1)$ & $60(32.1)$ & & No fibrosis & $52(27.8)$ & $31(16.6)$ & \\
\hline Non-alcoholic fatty liver disease & $8(4.3)$ & $8(4.3)$ & & Portal fibrosis & $14(6.8)$ & $18(8.8)$ & \\
\hline No risk factors & $46(24.6)$ & $52(27.8)$ & & Fibrous speta & $15(8.0)$ & $15(8.0)$ & \\
\hline Other & $9(4.8)$ & $12(6.4)$ & & Nodular Formation/Incomplete Cirrhosis & $7(3.7)$ & $3(1.6)$ & \\
\hline NA & $9(4.8)$ & $11(5.9)$ & & Cirrhosis & $40(21.4)$ & $44(23.5)$ & \\
\hline Neoplasm histologic grade, $n(\%)$ & & & $<0.01$ & NA & $76(40.6)$ & $95(50.8)$ & \\
\hline Grade 1 & $44(23.5)$ & $18(9.6)$ & & Hepatic inflammation, $\mathrm{n}(\%)$ & & & 0.029 \\
\hline Grade 2 & $113(60.4)$ & $86(45.9)$ & & None & $74(39.6)$ & $55(29.4)$ & \\
\hline Grade 3 & $40(21.4)$ & $90(48.1)$ & & Mild & $53(28.3)$ & $54(28.8)$ & \\
\hline Grade 4 & $4(2.1)$ & $8(4.1)$ & & Severe & $13(6.9)$ & $7(3.7$ & \\
\hline NA & $3(1.6)$ & $4(2.1)$ & & NA & $64(34.2)$ & $90(48.1)$ & \\
\hline Years to last follow up, median (IQR), years & $0.9(1.81)$ & $0.73(2.29)$ & 0.06 & & & & \\
\hline
\end{tabular}

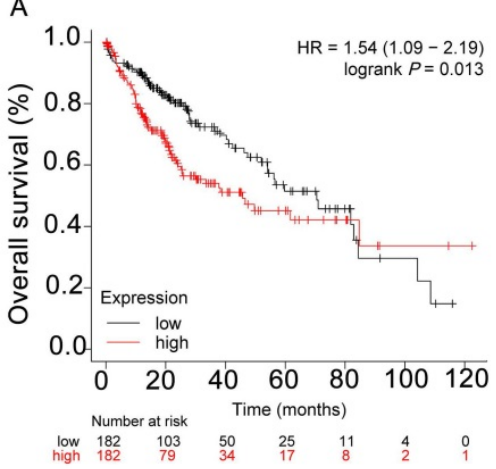

C

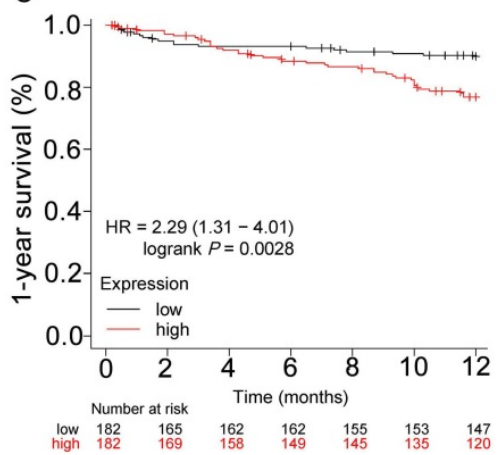

B

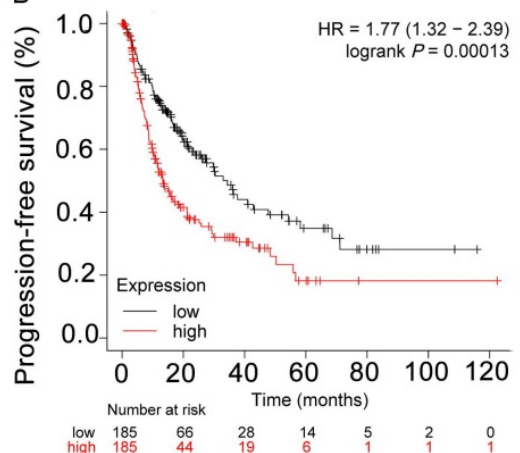

D

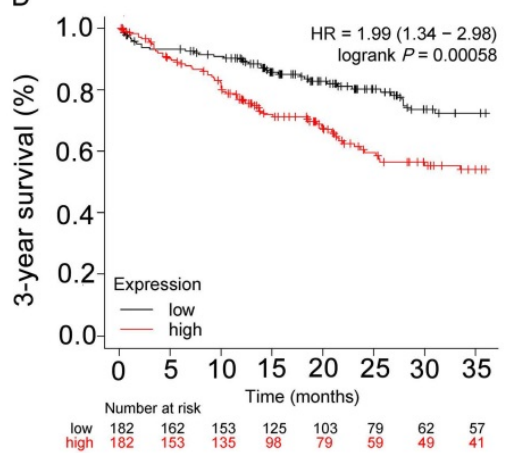

$\mathrm{E}$

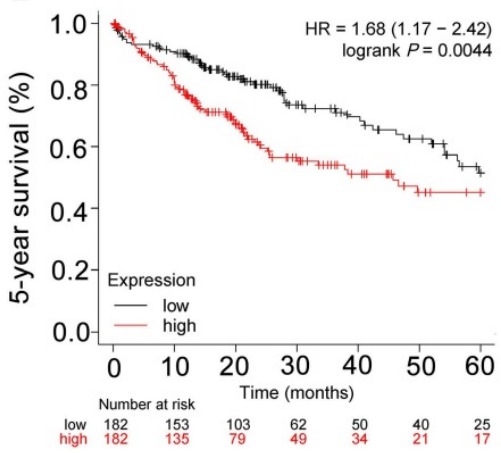

Figure 5. Overexpression of CENPF predicted worse prognosis in HCC patients (Kaplan-Meier plotter). The OS (A), PFS (B), 1-year (C), 3-year (D) and 5-year (E) survival curves comparing patients with high (red) and low (black) CENPF expression in HCC were plotted using Kaplan-Meier plotter database at the threshold of $P$-value $<0.05$. 

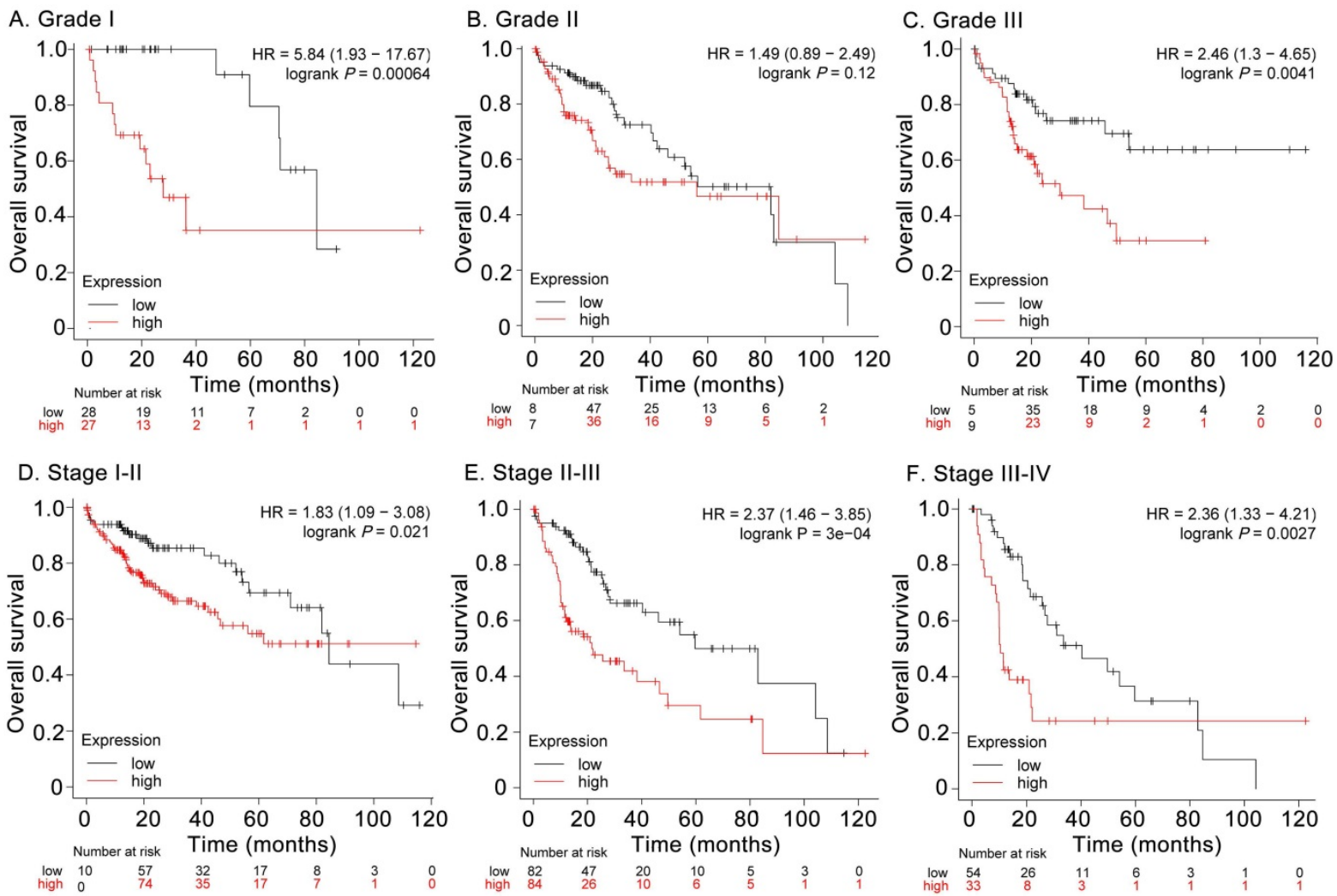

Figure 6. Overall survival of $H C C$ patients grouped by CENPF median in different grades (A, B, C) and stages (D, E, F).

Besides, we conducted subgroup survival analysis in different population. As shown in Figure S1, high expression of CENPF was significantly associated with worse OS in HCC patients without hepatitis virus infection $(\mathrm{HR}=1.87, \log \operatorname{rank} P=$ 0.0073 , Figure S1B). Overexpression of CENPF was significantly and negatively associated with OS both in men and women of $\mathrm{HCC}(\mathrm{HR}=1.93, \log \operatorname{rank} P=$ 0.0038 and $\mathrm{HR}=2, \log$ rank $P=0.017$, respectively, Figures S1C and S1D). CENPF was a risk factor for OS in Asian patients with $\mathrm{HCC}(\mathrm{HR}=4.26, \log$ rank $P=7 \mathrm{E}-06$, Figure S1F) and in HCC cases with alcohol consumption $(\mathrm{HR}=1.97, \log \operatorname{rank} P=0.0036$, Figure $\mathrm{S} 1 \mathrm{G})$.

\section{Inhibition of CENPF expression affects the proliferation, migration, and apoptosis of HCC}

Firstly, human HCC samples were used to confirm the expression of CENPF. According to the screening criteria for PPFE samples of HCC patients, 5 HCC tissues and 5 paired adjacent non-cancerous samples of PPFE were selected for IHC. As result, CENPF protein is over-expressed in human HCC tissues, compared with paired adjacent non-cancerous samples. (Figure 7A) According to The Human Protein Atlas database, CENPF was also high expressed in HepG2 cells. (Figure 2G) Thus, CENPF siRNAs (siCENPF) was constructed to investigate the role of CENPF in human HepG2 cells. The inhibition efficiency of siCENPF was more than $60 \%$ at RNA and protein levels for CENPF, compared with siNC. (Figures 7B-C) In CCK-8 cell proliferation experiment, down-regulation of $C E N P F$ significantly inhibited cell proliferation in human HepG2 cells at 1 hour (Figure 7D) and cell mobility at 24 hour (Figure 7E). Down-regulation of CENPF significantly inhibited cell migration (Figures 7F-G) and cell invasion (Figures 7H-I) in human HepG2 cells. Additionally, flow cytometry analysis showed that down-regulation of CENPF slightly promoted cell apoptosis in human HepG2 cells (27.2\% vs $31.8 \%$, Figure 7J).

\section{GSEA outlines underlying roles of CENPF in oncogenic signaling pathway}

To explore the potential molecular mechanisms by which CENPF alters tumor development and progress, GSEA enrichment analysis based on TCGA was utilized to analysis the gene expression profiles of CENPFlow and CENPFhigh expression in HCC specimens. According to GSEA analysis of KEGG pathways, HCC with CENPFigh was mainly involved in cell cycle, ubiquitin mediated proteolysis, and oocyte meiosis, etc. (Figure 8A, and Table S2) The top 6 enrichment pathways and related genes were circular visualization and listed as Figure 8A. The normalized enrichment scores and $P$-values of GSEA analysis were figured out in Figure 8B. 
A

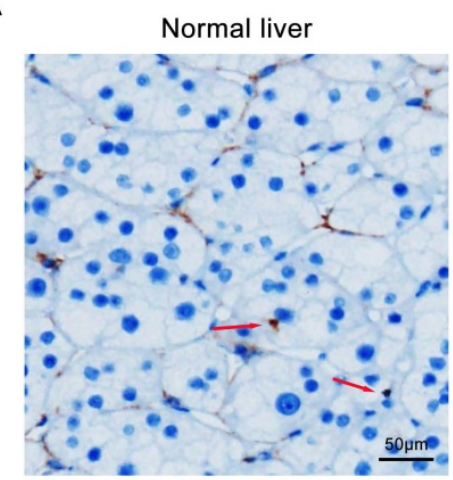

E
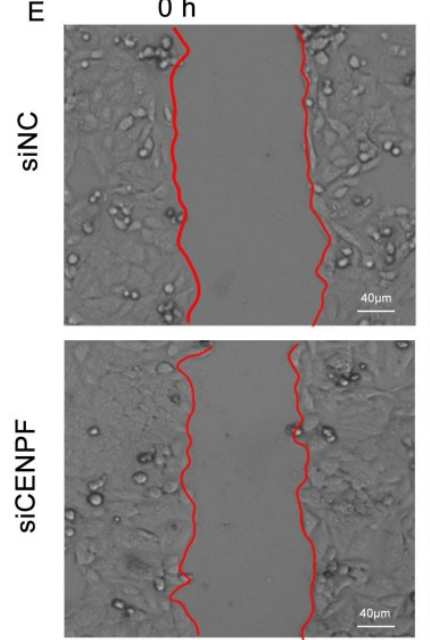

$\mathrm{H}$

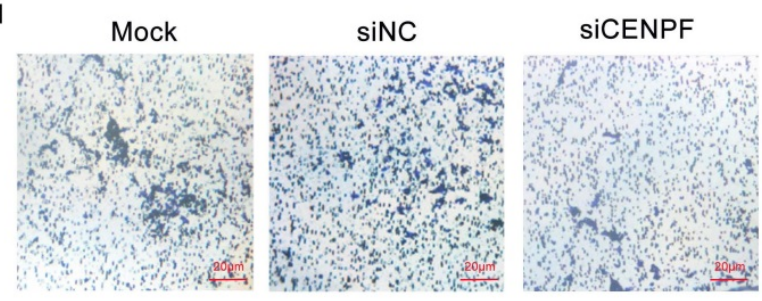

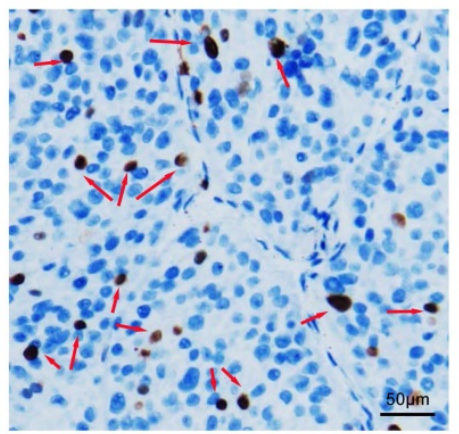

HCC liver

B

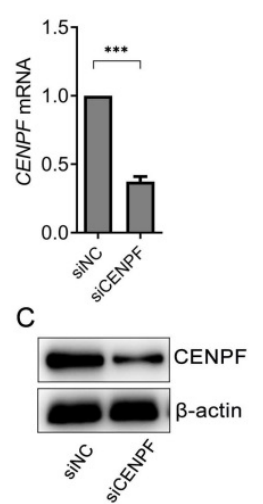

F

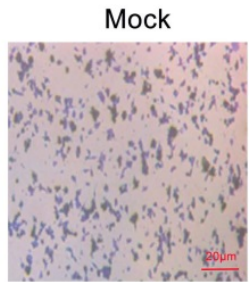

siNC
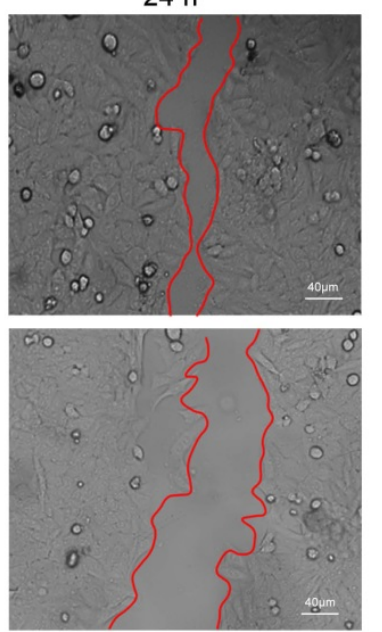

G
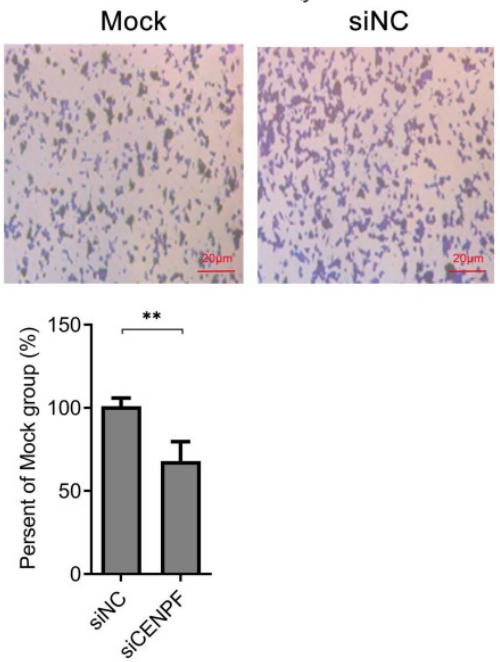

D

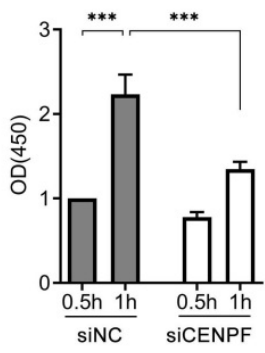

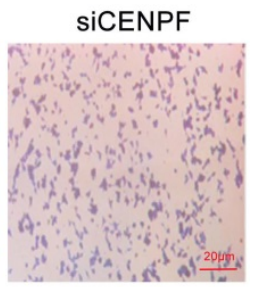
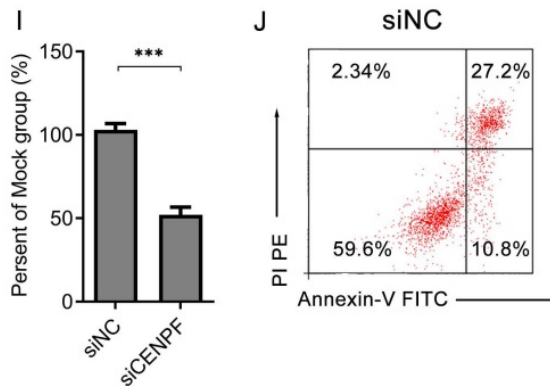

siCENPF

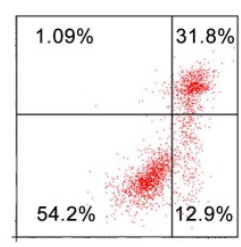

Annexin-V FITC

Figure 7. The expression of CENPF is involved in the cell proliferation and migration of HCC. (A) Immunohistochemistry of CENPF in human HCC samples; Blue represents hematoxylin (nucleus) and dark brown represents CENPF protein. The red arrows denote the expression of CENPF protein. (B) qRT-PCR and (C) Western blotting for CENPF expression in HepG2 cells after cells were transfected with homo-CENPF siRNA (siCENPF) or contorl siRNA (siNC) for 48 hours. $\beta$-actin molecular weight: 42 kDa, CENPF molecular weight: $330 \mathrm{kDa}$. (D) Cell Counting Kit-8 experiment for cell proliferation assay. (E) Wound healing assay for cell mobility investigation; The white line reflects the migration ability of HepG2 cells transfected with siCENPF or siNC for 24 hours. (F) Cell metastatic ability assay of HepG2 cells transfected with siCENPF or siNC for 24 hours. (G) Percentage of migratory cells compared to mock group. $(\mathrm{H})$ Cell invasion assay of HepG2 cells transfected with siCENPF or siNC for 24 hours. (I) Percentage of invasive cells compared to mock group. (J) Flow cytometry analysis of apoptosis of cells using Annexin-V/PI kit.

\section{The CENPF may participate in cell cycle regulation and MAPK pathway in HCC}

Furthermore, integrated GSEA enrichment analyses of KEGG pathway and Hallmark description were carried out to declare that cell cycle, G2/M checkpoint, mitotic spindle, and E2F targets were identified as the significant signatures affected by $C E N P F$, indicating that CENPF might affect cell cycle by interacting with $\mathrm{G} 2 / \mathrm{M}$ checkpoint, E2F targets, and mitotic process. (Figure 9A, and Tables S2-S3) It was reported that E2F1, a member of E2Fs family, was an pivotal transcription factor of CENPF in NCI-60 cell line [53], and E2F1 played an important role in G2/M transition and cell cycle regulation[54, 55]. Thus, we focused on E2F1 in HCC. Venn graph analysis demonstrated that 9 genes (BUB1, CDK1, TTK, CCNB2, PLK1, CDC27, SMC1A, ESPL1, and $A B L 1)$ were co-expressed in these three groups. (Figure 9B) By screening the correlation, expression data and prognosis information of these nine genes and E2F1, we found that CDK1 and E2F1 were 
evidently upregulated in HCC (Figure 9D, all $P<0.05$ ) compared with normal tissues, and significantly positively correlated with the expression of CENPF (Figure 9C, all $P<0.01$ ). The overexpression of $C D K 1$ and E2F1 also led to poor survival prognosis of HCC patients (Figure 9E, all $P<0.01$ ). Thus, CENPF-E2F1/CDK1 pathway mediating abnormal cell division in cell cycle might be a indispensable process in hepatocellular carcinoma. As listed in Figure 9F and Table S4, GSEA enrichment analysis of Biocarta description revealed that overexpression of CENPF was mostly involved in MAPK mediated inflammatory signaling pathway in the development and progress of HCC.
A.

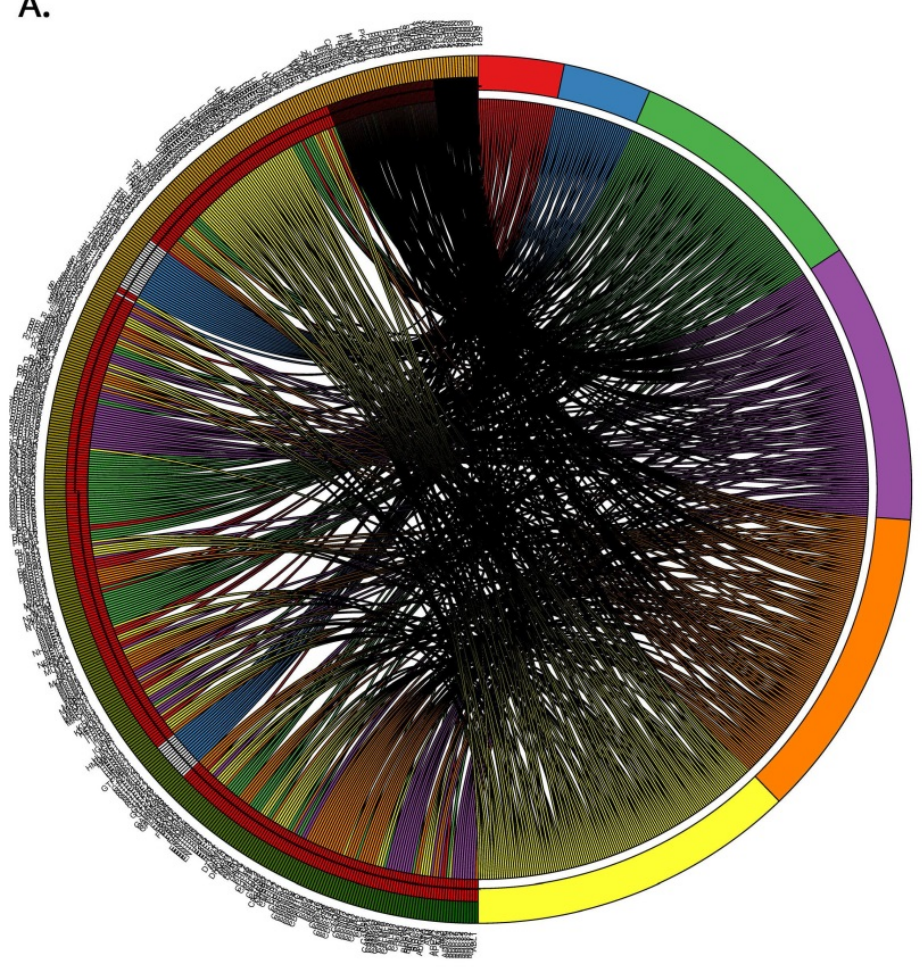

Left side:

Total frequency of genes in pathways

4.0

1.0

$P$ value

0.006

0.00

Right side: KEGG pathway terms

Base excision repair (34)

Basal transcription factors (35)

Pyrimidine metabolism (98)

Oocyte meiosis(111)

Cell cycle (124)

Ubiquitin mediated proteolysis (130)

B.

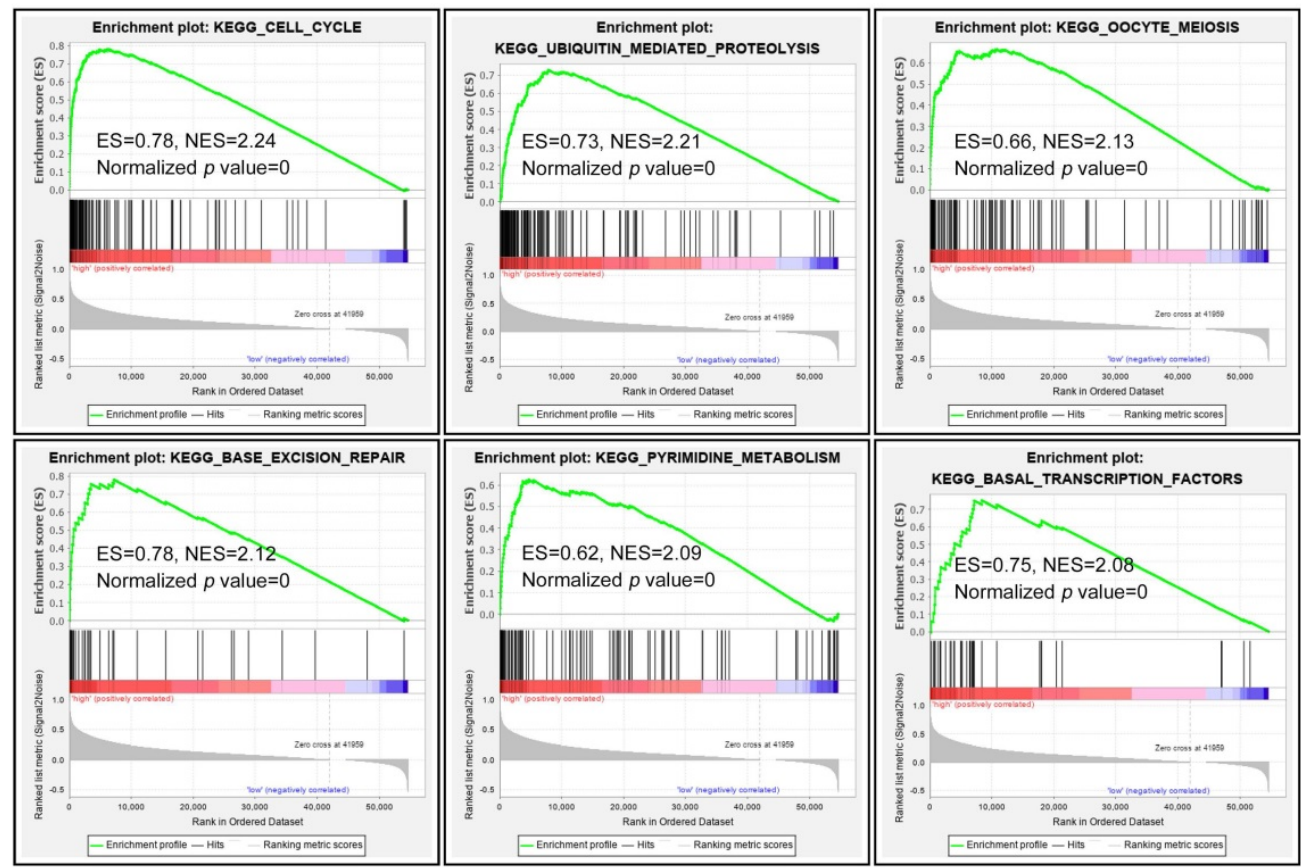

Figure 8. GSEA enrichment analysis of KEGG pathways in HCC samples with CENPFhigh versus CENPFlow. (A) Top 6 enrichment KEGG pathways and related genes; (B) Normalized enrichment scores and P-values of top 6 enrichment KEGG pathways. 
A
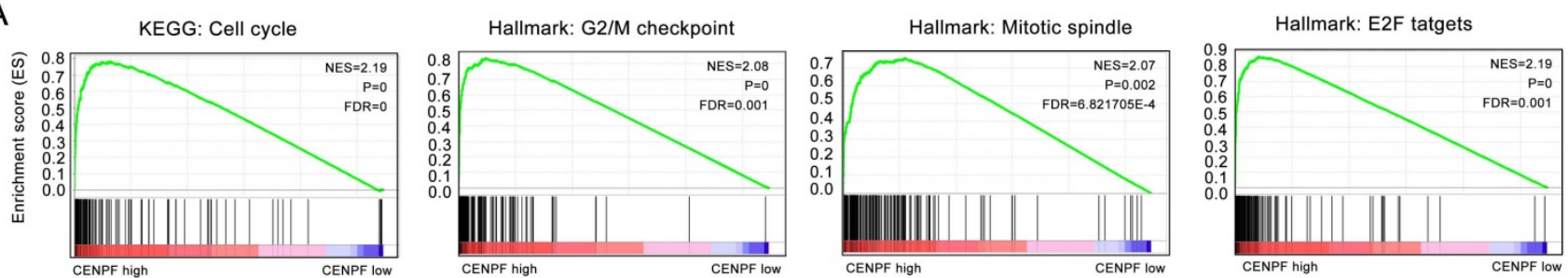

B

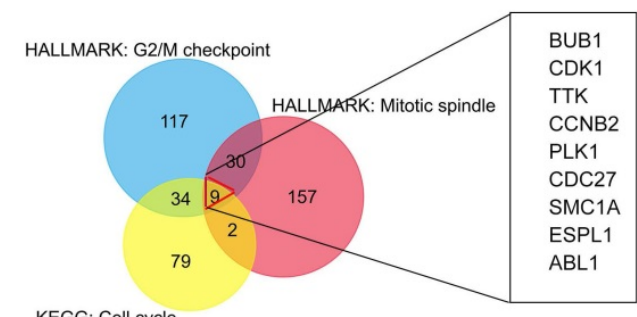

D
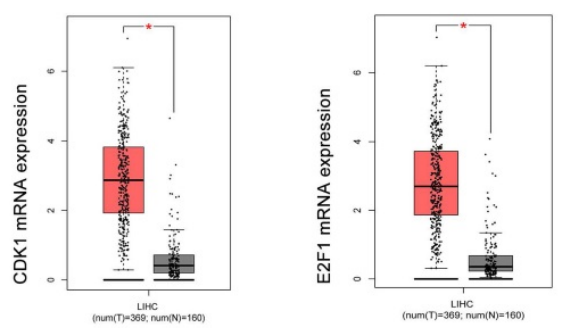

$\mathrm{F}$

\begin{tabular}{clcc}
\hline Rank & BIOCARTA description & NES & P value \\
\hline 1 & MAPK Pathway & 2.07 & $1.00 \mathrm{E}-10$ \\
2 & MAL Pathway & 2.03 & $1.10 \mathrm{E}-09$ \\
3 & HIV-I Nef Pathway & 2.03 & 0.000001 \\
4 & G2 Pathway & 2.02 & $1.10 \mathrm{E}-09$ \\
5 & TNFR1 Pathway & 2.02 & $1.01 \mathrm{E}-07$ \\
6 & VEGF Pathway & 2.02 & $1.10 \mathrm{E}-09$ \\
7 & RAS Pathway & 2.01 & $1.00 \mathrm{E}-07$ \\
8 & VDR Pathway & 2.00 & $1.11 \mathrm{E}-08$ \\
9 & FAS Pathway & 1.99 & $1.01 \mathrm{E}-07$ \\
10 & IGF1R Pathway & 1.97 & 0.00005 \\
\hline
\end{tabular}

C

E

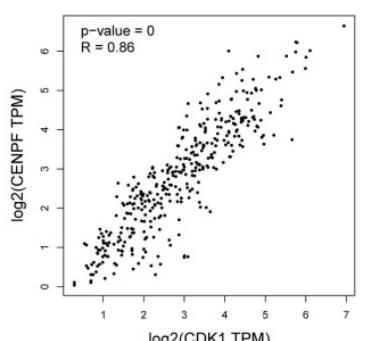

CDK1

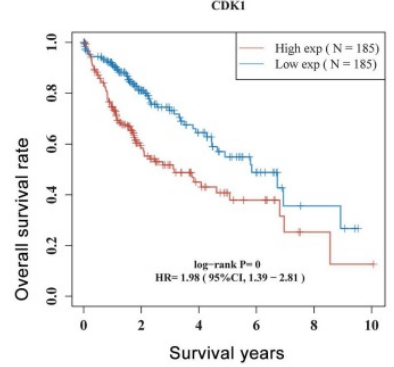

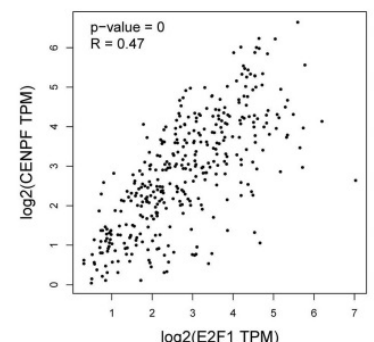

E2F1

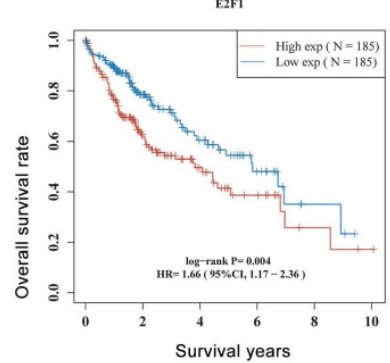

Figure 9. The CENPF may be involved in cell cycle and MAPK signaling pathway in HCC. (A) GSEA revealed that high CENPF expression was positively correlated with cell cycle pathway, G2/M checkpoint, mitotic spindle, and E2F targets. (B) Venn graph showed that BUB1, CDK1, TTK, CCNB2, PLK1, CDC27, SMC1A, ESPL1, and ABL1 were overlapped in the three groups. (C) Correlation of CDK1, E2F1 and CENPF expression in hepatocellular carcinoma. (D) Expression of CDK1 and E2F1 in hepatocellular carcinoma and normal liver tissues. (E) Prognostic analysis of CDK1 and E2F1 in hepatocellular carcinoma by GEPIA. (F) Top 10 GSEA enrichment analysis of Biocarta description.

\section{Signature analysis of immune infiltration in HCC by CIBERSORTx}

The expression matrix from TCGA datasets contains 424 HCC tissues divided to three groups, including 50 normal liver samples, 187 CENPFlow, and 187 CENPFigh HCC samples by median cutoffs, and normalized by 'Limma' packages. CIBERSORTx was applied to screen the 22 cell types potentially involved in the occurrence and development of HCC with low and high expression of CENPF. $\mathrm{P}<0.05$ was considered as statistically significant. Then, the filtered information including 12 normal liver samples, 42 HCC samples with CENPF low expression and 66 HCC samples with CENPF high expression was considered for further analysis. The immune cell types of each sample in forms of relative percentage (Figure 10A), and expression level (Figure 10B) were shown as heatmaps. The correlation between the 22 immune cell populations in HCC samples was shown in the Figure 10C. The principal component analysis (PCA) revealed that there was significantly different in immune infiltration between normal liver and HCC samples, but not obvious difference was found between the HCC with low CENPF expression group and the HCC with high CENPF expression group. (Figure 10D) It might suggest that the immune heterogeneity of the CENPFlow and CENPFhigh was not significant. Lastly, the immune enrichment fraction was significantly different in normal, 187 CENPFlow, and 187 CENPFhigh samples. (Figure 10E) As results in B cell population, 
the immune enrichment fraction of naïve B cells and plasma cells in HCC with CENPFhigh group were much lower than that in HCC with CENPFlow group or normal tissues. Otherwise, the immune enrichment index of memory B cells in HCC and normal group were all very low, even could not be detected. In T cell population, $\mathrm{T}$ regulatory cells $\left(\mathrm{T}_{\mathrm{reg}}\right)$, and follicular helper T cells were manifestly enriched in HCC with CENPFhigh, compared with HCC with CENPFlow or normal ones. However, there was no difference for CD8 and CD4 T cells. In NK cell population, the immune enrichment score of activated NK cells in HCC with CENPFhigh was slightly higher than that in
HCC with CENPFlow, but the difference was not significant compared with normal tissues. Moreover, the immune enrichment score of monocytes, macrophages M2 in HCC with CENPFhigh than that in HCC with CENPFlow or normal tissues. But for macrophages M0, the immune enrichment score gradually increased in the normal, CENPFlow, and CENPFhigh groups. In granulocytes group, the immune enrichment score of resting mast cells in HCC with CENPFhigh or CENPFlow were higher than that in normal tissues. But for activated mast cells and eosinophils, the scores were all very low, even could not be detected.

A

Type •normal liver ( $\mathrm{n}=12) \bullet \mathrm{HCC}$ with CENPF low $(\mathrm{n}=42) \quad$ HCC with CENPF high $(\mathrm{n}=66)$
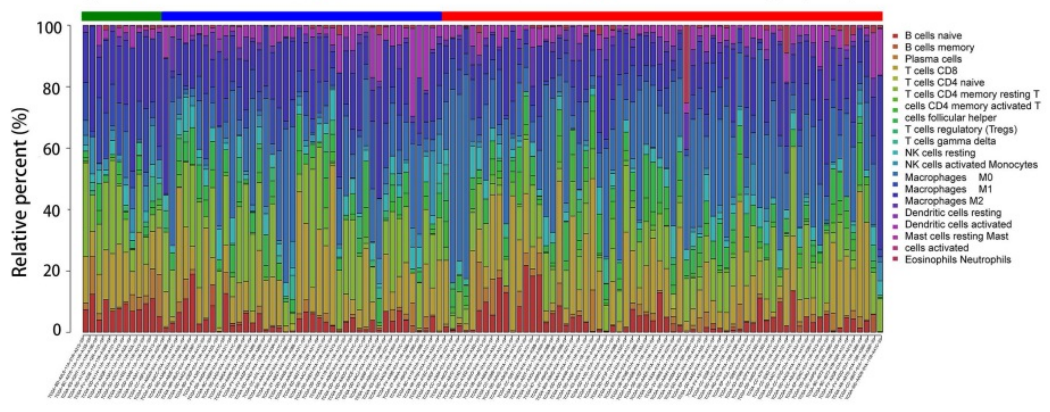

B

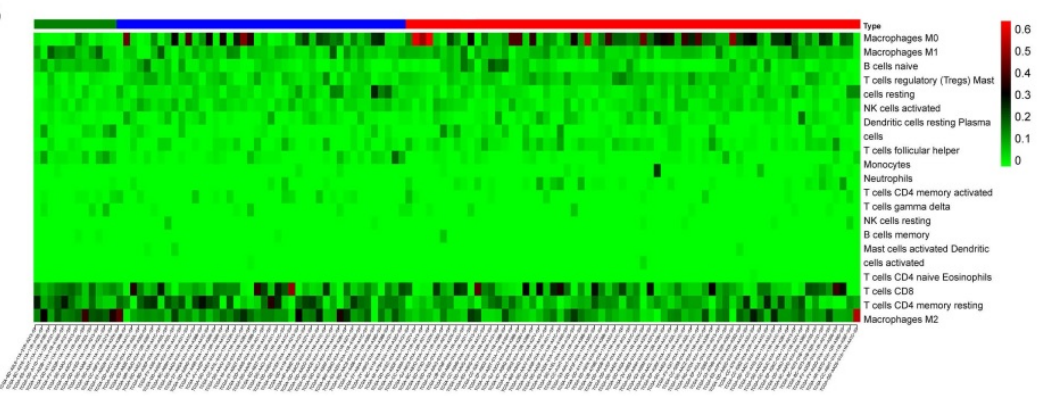

$E$
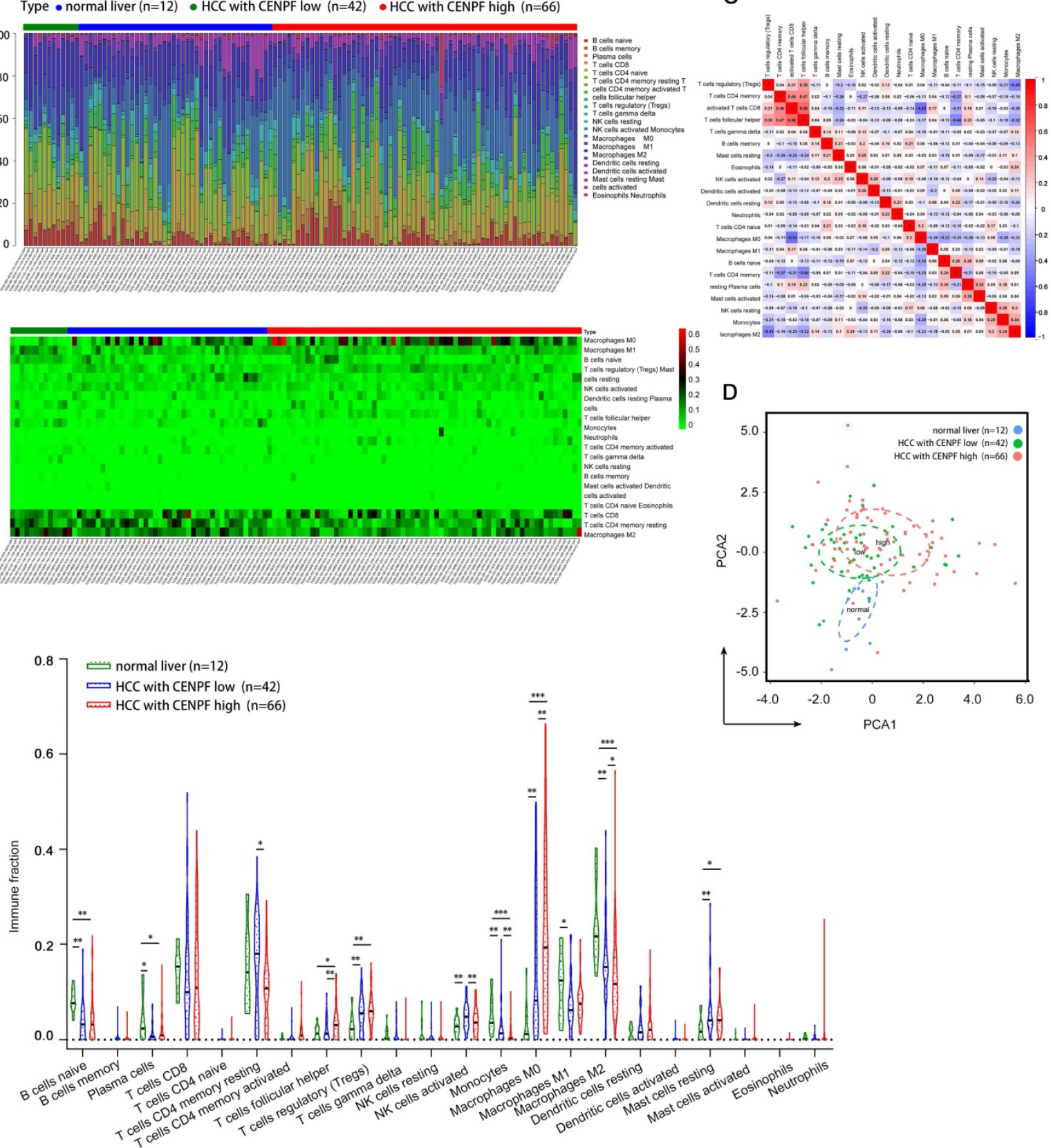
of 22 types of immune cells in each sample. (B) The difference of immune cells infiltration for three groups visualized by heatmap. The correlation between the 22 immune cell populations(C) and PCA analysis (D), and immune fraction (E) in HCC samples. 

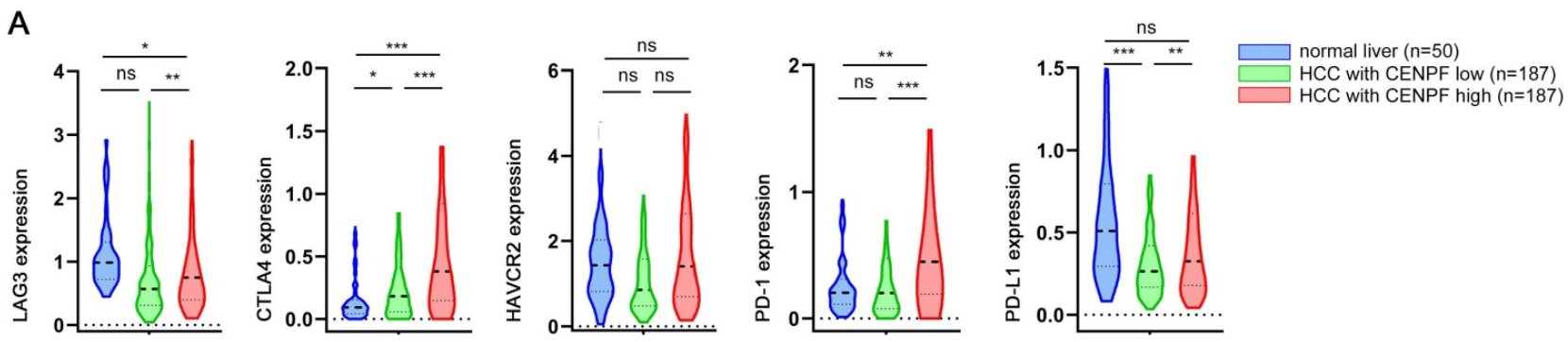

B
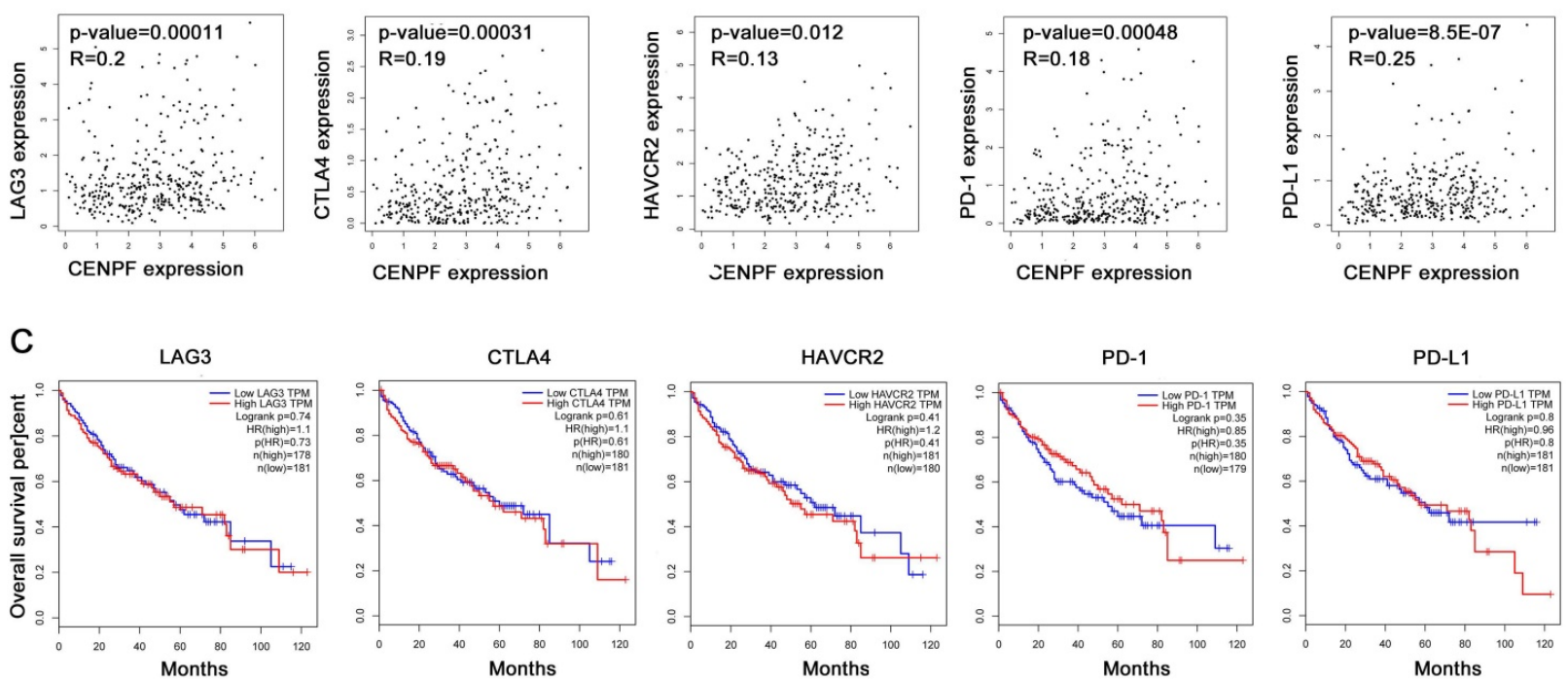

D

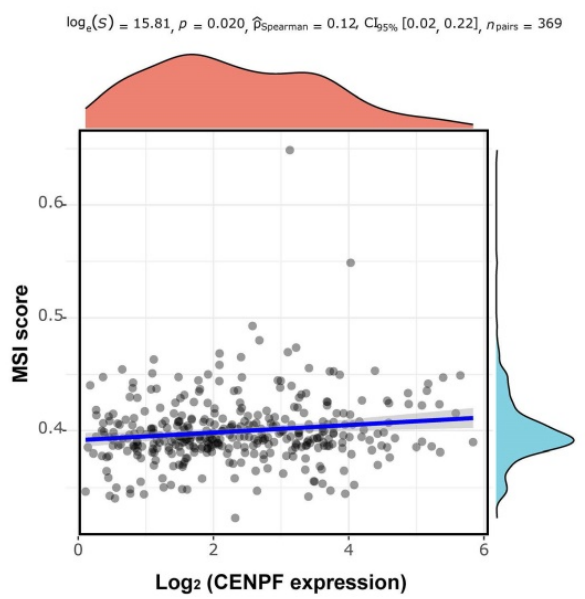

$\mathrm{E}$

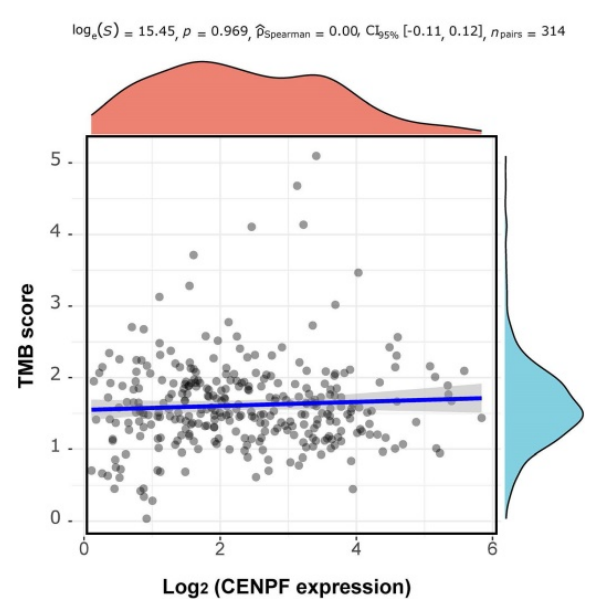

Figure 11. Objective to screen the expression, correlation and prognosis of CENPF with these five genes (LAG3, CTLA4, HAVCR2, PD-1, and PD-L1), MSI and TMB in hepatocellular carcinoma. (A) The expression of these five genes in CENPFhigh, CENPFlow, and normal liver tissues. (B) The correlation between CENPF and five genes in HCC. (C) Survival analysis of the five genes in HCC. Correlation analysis of CENPF expression and MSI(D) and TMB(E) in HCC. The horizontal axis in the figure represents the expression distribution of the gene, and the ordinate is the expression distribution of the TMB/MSI score. The density curve on the right represents the distribution trend of the $\mathrm{TMB} / \mathrm{MSI}$ score; the upper density curve represents the distribution trend of the gene; the top side. The value represents the correlation $\mathrm{p}$ value, correlation coefficient and correlation calculation method.

In addition, the mRNA levels of LAG3, CTLA4, HAVCR2, PD-1, and PD-L1 in HCC samples, which were closely related to tumor immunosuppression or tolerance, were shown in Figure 11. The expression of LAG3, CTLA4, PD-1, and PD-L1 were upregulated in HCC with CENPFhigh, compared to HCC with CENPFlow. (Figure 11A) Moreover, the correlation between the expression of these five genes and CENPF were further explored in HCC patients. The expression of LAG3, CTLA4, HAVCR2, PD-1, and $P D$-L1 were not markedly positive correlated with CENPF (Figure $11 \mathrm{~B}$, all $\mathrm{R}<0.75$ ), and there was no significant correlation between the expression of these five genes and the overall survival rate in HCC 
patients (Figure 11C, all $P>0.05$ ). Lastly, the correlation between CENPF expression and MSI or TMB were also screened. It showed that the expression of CENPF was not significantly related to MSI or TMB in HCC (Figures 11D-E, all the value of Spearman's correlation coefficient, $\mid \rho$ spearman $\mid$, is close to 0 ).

\section{Discussion}

Numerous studies have manifested that CENPF is not only involved in cell proliferation but also in tumorigenesis [26-28]. In the past few years, evidence has accumulated that abnormal expression or activation of CENPF is a common phenomenon in malignant tumors, and there is a significant correlation between CENPF and tumorigenesis or progression in cancer patients, including HCC [27, 28], prostate cancer, breast cancer [29], and other tumors [30], which has been partially confirmed. Aytes A, et al. reported that CENPF has been identified as a major co-regulator of prostate cancer and a poor prognostic indicator of survival and metastasis [31]. However, the patterns of expression and the exact roles of CENPF in HCC patients are not yet well known, and the molecular mechanism and the functions of CENPF remain undefined. The purpose of this study was to systematically investigate the expression alteration, prognostic values, correlation, and potential functions of CENPF in HCC.

Integration of multiple arrays has been viewed as a more reasonable method to improve detection capabilities and enhancing the reliability of results than single array analysis [56]. In present study, we have gained insight into gene expression of CENPF through analyzing HCC datasets from GEO, TCGA and Oncomine. Moreover, protein expression of CENPF in HCC tissues and their associated normal tissues were also screened in The Human Protein Atlas by IHC staining. The staining intensity of CENPF antibody was mainly in HCC tissues and weakly positive in normal adjacent tissues. The information from GEO datasets indicated that CENPF was upregulated in $\mathrm{HCC}$, including primary and metastasis with lung or adrenal gland tissue, compared with normal ones. But no significant difference was found between primary HCC and HCC with lung or adrenal gland metastasis tissues. Consistent with the results, CENPF was found to be significantly upregulated in cirrhosis, liver cell dysplasia and HCC tumors compared with normal livers. Additionally, Sung WK et al. reported that the expression of CENPF is significantly higher in HCC tissues than that in cirrhosis [57]. Wurmbach E et al. reported that mRNA level of CENPF is significantly upregulated in HCC tissues than that in cirrhosis, in liver cell dysplasia and in normal livers. But no significant difference was found between cirrhosis or liver cell dysplasia and normal livers. [58] Based on TCGA datasets, uni-cox and mult-cox analysis was performed to figure out that the CENPF expression and pTNM stage might be independent prognostic factors in HCC patients. Hence, we assumed that CENPF may be emerging as a biomarker to distinguish HCC from normal livers, but further studies should be conducted to discriminate benign liver lesions from normal livers.

Then, Kaplan-Meier analyses of CENPF were performed to show that high expression levels of CENPF in tumor were significantly associated with the deterioration of OS and PFS in HCC patients. Overexpression of CENPF were associated with poor 1-year, 3-year and 5-year OS, and it seemed that hazard ratio (HR) of CENPF overexpression associated with OS was more obvious in the early years of HCC. In subgroup analyses, overall survival comparison in population of Asian, both male and female and without hepatitis virus infection were significantly associated with worse OS in HCC patients with CENPF overexpression (Figure S1). It indicated that this may provide a clue to the prognosis or treatment of HCC patients with high expression of CENPF and non-hepatitis virus infection. A series of publication reinforced our results. Dai $Y$ et al. showed that upregulation of CENPF in HCC is positively associated with serum AFP, venous invasion, advanced differentiation stage and a shorter overall survival, and overexpression of CENPF was a risk factor for the prognosis of HCC. Inhibition of CENPF expression could reduce the ability of cell proliferation, migration, and tumor formation in nude mice, and block the cell cycle at G2/M checkpoint. [27] Kim H E et al. reported that gene amplification of CENPF is closely related to tumor formation and development, and it plays a role as a cancer-driver gene in human cancers [59]. A recent study identified that overexpression of CENPF and/or Lymphoidspecific helicase $(L S H)$ are correlated with shorter overall survival and higher cumulative recurrence rates in HCC patients. LSH may promote the development of HCC by transcriptional regulation of CENPF expression [28]. Generally, our findings suggested that CENPF was overexpressed in HCC and might play a critical role in driving HCC tumorigenesis. Functionally, as a member of centromere protein family, CENPF might exert a pivotal role through regulating chromosome segregation during mitosis cell cycle at G2/M checkpoint. 
For the purpose of verifying the above results, immunohistochemistry for the human samples was performed to confirm that the expression of CENPF was upregulated in HCC patients, compared with paired adjacent tissues. Furthermore, a down-regulation system of CENPF, siRNA, was constructed to probe the effect of CENPF on the function of hepatoma cells in the cell line of human HepG2 cells. The results asserted that down-regulation of CENPF inhibited the proliferation, migration, and invasion of HCC cells, and slight promoted the apoptosis of HCC cells (27.2\% vs $31.8 \%$, Figure $7 \mathrm{~J})$. It suggested that CENPF might play a crucial role in promoting the proliferation, migration, invasion, and inhibiting apoptosis of HCC cells during the progression or deterioration of HCC.

Then, the Gene-set enrichment analysis (GSEA) of KEGG pathway from TCGA datasets in current study pointed out that CENPFhigh was mostly enriched in cell cycle pathway, closely related to the occurrence of a variety of tumors. Meanwhile, according to Hallmark description and Biocarta description, we found that CENPF was closely related to E2F1 and CDK1in the regulation of cell cycle, especially during G2 / M transition of mitosis. In addition, CENPF might also participate in MAPK signaling pathway in the development and progression of HCC. Therefore, our viewpoint threw light on the growing evidence regarding CENPF interactive genes and their associated signaling pathways, which might provide clues for the development of CENPF-mediated targeted therapy. However, further molecular mechanism, pharmaceutical and clinical studies are needed to confirm this prediction. Unfortunately, further experimental research for exploring the latent carcinogenic mechanism of CENPF in the occurrence and development of HCC could not be carried out by us. And, we did not have our own follow-up information of HCC patients.

As far as we know, HCC is tumor with primarily resistance to chemotherapeutic drugs [18]. Although novel biomarkers and multiple molecular mechanisms of HCC have been studied, effective drugs for the treatment of HCC are still scarce [60]. As one of hallmarks of cancer, immune microenvironment and immunotherapy has attracted much attention in the research of liver cancer in recent years. The presence of lymphocytic infiltration in tumors has been considered to be one of the prerequisites for immunotherapy [61]. In this paper, immune cell infiltration analysis of HCC with CENPF high and low expression was constructed by CIBERSORTx to reveal that the immune cell types were significantly different in HCC samples with CENPFhigh, compared with normal liver samples, and HCC samples with CENPFlow. As results, $T$ regulatory cells $\left(\mathrm{T}_{\text {reg }}\right)$, follicular helper $\mathrm{T}$ cells, macrophages $\mathrm{M} 0$, and resting mast cells were manifestly enriched in HCC with CENPFigh, compared with CENPFlow or normal ones. While monocytes, macrophages M1 and M2, activated NK cells were downregulated in CENPFigh group, compared with CENPFlow group. The proportion B cells was low, and most of them were in the state of naïve B cells. The result declared that immunosuppressive microenvironment has played predominant roles in the occurrence and development of HCC with CENPF high expression, such as high immune infiltration score of $\mathrm{T}_{\text {reg, }}$, resting mast cells, and down-regulation of monocytes and neutrophils.

A variety of immunosuppressive mechanisms have been found in tumor microenvironment, including PD-1 / PD-L1, CTLA-4, PD-L1, LAG3, etc. Thus, five genes closely related to immunotherapy were investigated in this study. As reported, LAG-3 was confirmed to be involved in the proliferation of $\mathrm{T}$ cells in vitro and in vivo. Costimulatory molecules, including LAG3, PD-1, CTLA4, and TIGIT, are expressed on dysfunctional or depleted $\mathrm{T}$ cells in chronic viral infection and tumor [62]. Tumor growth was inhibited in LAG3 knockout mice [63]. As results, LAG-3, CTLA-4, PD-1, and PD-L1 were enriched in in HCC samples with CENPFigh, compared with CENPFlow or normal groups, but no significant positive correlation was found between expression of LAG3, CTLA4, HAVCR2, PD-1, and PD-L1 and the expression of CENPF. The OS of the five gene was not statistically significant in HCC patients. Therefore, HCC patients with CENPFhigh group may not benefit from immunotherapy targeting these five genes.

The incidence of MSI is high in a variety of tumors, such as endometrial carcinoma, intestinal adenocarcinoma, gastric adenocarcinoma and so on. As a molecular marker of tumor cells, MSI-H plays an important role in predicting the antitumor effect of PD-1 / PD-L1 inhibitors [64, 65]. Additionally, clinical studies have shown that TMB is closely related to the efficacy of existing PD-1 / PD-L1 inhibitors. Patients with high TMB are more likely to benefit from immunotherapy. TMB, as an independent factor of immune response to PD-L1, has a positive linear correlation with immune response, and its scope of application is not limited by pathological types [66]. However, the expression of CENPF was not significantly related to MSI or TMB in HCC with the CENPF expression.

Collectively, we speculated that immunotherapy targeting these five target genes (especially including 
PD-1 / PD-L1, MSI, and TMB) has no obvious effect in patients with HCC with high CENPF expression. However, several immunosuppressive regents, including $\mathrm{T}_{\text {reg, }}$ resting mast cells, monocytes, and neutrophils, might be viewed as potential therapeutic targets for the immunotherapy of HCC patients with CENPFhigh.

Eventually, based on current reports, a hypothesis was cautiously drawn that overexpression of CENPF contributed to unfavorable prognosis in HCC patients. Inhibition of CENPF expression might be conducive to alleviate the progression or treatment of HCC.

In summary, our study gave results that the mRNA and protein expression levels of CENPF were significantly upregulated in HCC. Aberrant expression of CENPF was associated with the clinical prognosis of HCC patients. The overexpression of CENPF was tightly connected to the mitosis of cancer cells and the occurrence and development of HCC. The results suggested that $C E N P F$ may serve as potential prognostic biomarkers and targets for HCC patients. Meanwhile, these results may be helpful for us to better understand the molecular mechanism of HCC and useful to develop tools for more accurate HCC prognosis and promote the development of CENPF-mediated therapeutic drugs for HCC. Evidently, further research on the basic molecular mechanism and clinical application of CENPF are still needed to improve the clinical value of $C E N P F$ as a prognostic indicator or therapeutic target for HCC.

\section{Supplementary Material}

Supplementary figures and tables.

http://www.jcancer.org/v12p2933s1.zip

\section{Acknowledgements}

This work was supported by National Natural Science Foundation of China (grant number 81600436 and 81970488).

\section{Data Availability}

Publicly available datasets analyzed in this study can be found as follows.

GEO: https://www.ncbi.nlm.nih.gov/geo/; TCGA: https://portal.gdc.cancer.gov/; Oncomine: https://www.oncomine.org; The Human Protein Atlas: https://www.proteinatlas.org.

\section{Author Contributions}

Yugang Huang conceived, designed, performed statistical analysis and wrote the paper. Xiaomin Su and Xianbin Tang supervised the research. Xiuwen Chen participated in the study design and performed the R/Bioconductor software; Li Wang provisioned useful suggestions in methodology. Tieyan Wang provisioned suggestions in figure preparation. Yugang Huang and Xiaomin Su performed the detection of human HCC samples and in vitro experiments. All authors read and approved the final version of the manuscript.

\section{Competing Interests}

The authors have declared that no competing interest exists.

\section{References}

1. Harris PS, Hansen RM, Gray ME, Massoud OI, McGuire BM, Shoreibah MG. Hepatocellular carcinoma surveillance: An evidence-based approach. World J Gastroenterol. 2019; 25: 1550-9.

2. Bray F, Ferlay J, Soerjomataram I, Siegel RL, Torre LA, Jemal A. Global cancer statistics 2018: GLOBOCAN estimates of incidence and mortality worldwide for 36 cancers in 185 countries. CA Cancer J Clin. 2018; 68: 394-424.

3. Lordick F. Hepatocellular carcinoma-united forces against a global killer. Ann Oncol. 2020; 31: 449-50.

4. Villanueva A. Hepatocellular Carcinoma. N Engl J Med. 2019; 380: 1450-62.

5. Rahib L, Smith BD, Aizenberg R, Rosenzweig AB, Fleshman JM, Matrisian LM. Projecting cancer incidence and deaths to 2030: the unexpected burden of thyroid, liver, and pancreas cancers in the United States. Cancer Res. 2014; 74: 2913-21.

6. Balogh J, Victor D, 3rd, Asham EH, Burroughs SG, Boktour M, Saharia A, et al. Hepatocellular carcinoma: a review. J Hepatocell Carcinoma. 2016; 3: 41-53.

7. Petrick JL, Kelly SP, Altekruse SF, McGlynn KA, Rosenberg PS. Future of Hepatocellular Carcinoma Incidence in the United States Forecast Through 2030. J Clin Oncol. 2016; 34: 1787-94

8. Omata M, Cheng AL, Kokudo N, Kudo M, Lee JM, Jia J, et al. Asia-Pacific clinical practice guidelines on the management of hepatocellular carcinoma: a 2017 update. Hepatol Int. 2017; 11: 317-70.

9. Zheng H, Pomyen Y, Hernandez MO, Li C, Livak F, Tang W, et al. Single-cell analysis reveals cancer stem cell heterogeneity in hepatocellular carcinoma. Hepatology. 2018; 68: 127-40.

10. Friemel J, Rechsteiner M, Frick L, Böhm F, Struckmann K, Egger M, et al. Intratumor heterogeneity in hepatocellular carcinoma. Clin Cancer Res. 2015; 21: 1951-61.

11. Cronin KA, Lake AJ, Scott S, Sherman RL, Noone AM, Howlader N, et al. Annual Report to the Nation on the Status of Cancer, part I: National cancer statistics. Cancer. 2018; 124: 2785-800.

12. Jemal A, Ward EM, Johnson CJ, Cronin KA, Ma J, Ryerson B, et al. Annual Report to the Nation on the Status of Cancer, 1975-2014, Featuring Survival. J Natl Cancer Inst. 2017; 109: 1-22.

13. Losic B, Craig AJ, Villacorta-Martin C, Martins-Filho SN, Akers N, Chen X, et al. Intratumoral heterogeneity and clonal evolution in liver cancer. Nat Commun. 2020; 11: 291-305.

14. De Toni EN, Schlesinger-Raab A, Fuchs M, Schepp W, Ehmer U, Geisler F, et al. Age independent survival benefit for patients with hepatocellular carcinoma (HCC) without metastases at diagnosis: a population-based study. Gut. 2020; 69: 168-76.

15. Allemani C, Matsuda T, Di Carlo V, Harewood R, Matz M, Nikšić M, et al. Global surveillance of trends in cancer survival 2000-14 (CONCORD-3): analysis of individual records for 37513025 patients diagnosed with one of 18 cancers from 322 population-based registries in 71 countries. Lancet. 2018; 391: 1023-75.

16. Zheng R, Qu C, Zhang S, Zeng H, Sun $\mathrm{K}, \mathrm{Gu}$ X, et al. Liver cancer incidence and mortality in China: Temporal trends and projections to 2030. Chin J Cancer Res. 2018; 30: 571-9.

17. Chen LT, Martinelli E, Cheng AL, Pentheroudakis G, Qin S, Bhattacharyya GS, et al. Pan-Asian adapted ESMO Clinical Practice Guidelines for the management of patients with intermediate and advanced/relapsed hepatocellular carcinoma: a TOS-ESMO initiative endorsed by CSCO, ISMPO, JSMO, KSMO, MOS and SSO. Ann Oncol. 2020; 31: 334-51.

18. Llovet JM, Montal R, Sia D, Finn RS. Molecular therapies and precision medicine for hepatocellular carcinoma. Nat Rev Clin Oncol. 2018; 15: 599-616.

19. Chen B, Garmire L, Calvisi DF, Chua MS, Kelley RK, Chen X. Harnessing big 'omics' data and AI for drug discovery in hepatocellular carcinoma. Nat Rev Gastroenterol Hepatol. 2020; 17: 238-51.

20. Nishida N, Kudo M. Oncogenic Signal and Tumor Microenvironment in Hepatocellular Carcinoma. Oncology. 2017; 93 Suppl 1: 160-4.

21. Ally A, Balasundaram M, al RCe. Comprehensive and Integrative Genomic Characterization of Hepatocellular Carcinoma. Cell. 2017; 169: 1327-41.e23.

22. Zhu Y, Yang J, Xu D, Gao XM, Zhang Z, Hsu JL, et al. Disruption of tumour-associated macrophage trafficking by the osteopontin-induced colony-stimulating factor-1 signalling sensitises hepatocellular carcinoma to anti-PD-L1 blockade. Gut. 2019; 68: 1653-66. 
23. Liu GM, Li XG, Zhang YM. Prognostic role of PD-L1 for HCC patients after potentially curative resection: a meta-analysis. Cancer Cell Int. 2019; 19: 22.

24. Zong Z, Zou J, Mao R, Ma C, Li N, Wang J, et al. M1 Macrophages Induce PD-L1 Expression in Hepatocellular Carcinoma Cells Through IL-1 $\beta$ Signaling. Front Immunol. 2019; 10: 1643-54.

25. Easom NJW, Marks M, Jobe D, Gillmore R, Meyer T, Maini MK, et al. ULBP1 Is Elevated in Human Hepatocellular Carcinoma and Predicts Outcome. Front Oncol. 2020; 10: 971-81.

26. Hussein D, Taylor SS. Farnesylation of Cenp-F is required for G2/M progression and degradation after mitosis. J Cell Sci. 2002; 115: 3403-14.

27. Dai Y, Liu L, Zeng T, Zhu YH, Li J, Chen L, et al. Characterization of the oncogenic function of centromere protein $\mathrm{F}$ in hepatocellular carcinoma. Biochem Biophys Res Commun. 2013; 436: 711-8.

28. Yang $X$, Miao BS, Wei $C Y$, Dong RZ, Gao PT, Zhang $X Y$, et al. Lymphoid-specific helicase promotes the growth and invasion of hepatocellular carcinoma by transcriptional regulation of centromere protein $\mathrm{F}$ expression. Cancer Sci. 2019; 110: 2133-44.

29. Sun J, Huang J, Lan J, Zhou K, Gao Y, Song Z, et al. Overexpression of CENPF correlates with poor prognosis and tumor bone metastasis in breast cancer. Cancer Cell Int. 2019; 19: 264-74.

30. Varis A, Salmela AL, Kallio MJ. Cenp-F (mitosin) is more than a mitotic marker. Chromosoma. 2006; 115: 288-95.

31. Aytes A, Mitrofanova A, Lefebvre C, Alvarez MJ, Castillo-Martin M, Zheng T, et al. Cross-species regulatory network analysis identifies a synergistic interaction between FOXM1 and CENPF that drives prostate cancer malignancy. Cancer Cell. 2014; 25: 638-51.

32. Lin SC, Kao CY, Lee HJ, Creighton CJ, Ittmann MM, Tsai SJ, et al. Dysregulation of miRNAs-COUP-TFII-FOXM1-CENPF axis contributes to the metastasis of prostate cancer. Nat Commun. 2016; 7: 11418-31.

33. Sealfon SC, Chu TT. RNA and DNA microarrays. Methods Mol Biol. 2011; 671:

34. Ritchie ME, Phipson B, Wu D, Hu Y, Law CW, Shi W, et al. limma powers differential expression analyses for RNA-sequencing and microarray studies. Nucleic Acids Res. 2015; 43: 47-59.

35. Shi J, Chen L, Chen Y, Lu Y, Chen X, Yang Z. Aldo-Keto Reductase Family 1 Member B10 (AKR1B10) overexpression in tumors predicts worse overall survival in hepatocellular carcinoma. J Cancer. 2019; 10: 4892-901.

36. Samur MK. RTCGAToolbox: a new tool for exporting TCGA Firehose data. PLoS One. 2014; 9: 106397-404.

37. Robinson MD, McCarthy DJ, Smyth GK. edgeR: a Bioconductor package for differential expression analysis of digital gene expression data. Bioinformatics. 2010; 26: 139-40.

38. Rhodes DR, Kalyana-Sundaram S, Mahavisno V, Varambally R, Yu J, Briggs $\mathrm{BB}$, et al. Oncomine 3.0: genes, pathways, and networks in a collection of 18,000 cancer gene expression profiles. Neoplasia. 2007; 9: 166-80.

39. Tang Z, Li C, Kang B, Gao G, Li C, Zhang Z. GEPIA: a web server for cancer and normal gene expression profiling and interactive analyses. Nucleic Acids Res. 2017; 45: 98-102

40. Zhang Z, Lin E, Zhuang H, Xie L, Feng X, Liu J, et al. Construction of a novel gene-based model for prognosis prediction of clear cell renal cell carcinoma. Cancer Cell Int. 2020; 20: 27.

41. Lin W, Wu S, Chen X, Ye Y, Weng Y, Pan Y, et al. Characterization of Hypoxia Signature to Evaluate the Tumor Immune Microenvironment and Predict Prognosis in Glioma Groups. Front Oncol. 2020; 10: 796.

42. Zeng D, Li M, Zhou R, Zhang J, Sun H, Shi M, et al. Tumor Microenvironment Characterization in Gastric Cancer Identifies Prognostic and Immunotherapeutically Relevant Gene Signatures. Cancer Immunol Res. 2019; 7: 737-50.

43. Xiong Y, Yuan L, Xiong J, Xu H, Luo Y, Wang G, et al. An outcome model for human bladder cancer: A comprehensive study based on weighted gene co-expression network analysis. J Cell Mol Med. 2020; 24: 2342-55.

44. Jeong SH, Kim RB, Park SY, Park J, Jung EJ, Ju YT, et al. Nomogram for predicting gastric cancer recurrence using biomarker gene expression. Eur J Surg Oncol. 2020; 46: 195-201.

45. Nagy Á, Lánczky A, Menyhárt O, Győrffy B. Validation of miRNA prognostic power in hepatocellular carcinoma using expression data of independent datasets. Sci Rep. 2018; 8: 9227-35.

46. Subramanian A, Tamayo P, Mootha VK, Mukherjee S, Ebert BL, Gillette MA, et al. Gene set enrichment analysis: A knowledge-based approach for interpreting genome-wide expression profiles. Proceedings of the National Academy of Sciences. 2005; 102: 15545-50.

47. Mootha VK, Lindgren CM, Eriksson K-F, Subramanian A, Sihag S, Lehar J, et al. PGC-1a-responsive genes involved in oxidative phosphorylation are coordinately downregulated in human diabetes. Nature Genetics. 2003; 34: 267-73.

48. Galon J, Costes A, Sanchez-Cabo F, Kirilovsky A, Mlecnik B, Lagorce-Pagès C, et al. Type, density, and location of immune cells within human colorectal tumors predict clinical outcome. Science. 2006; 313: 1960-4.

49. Newman AM, Steen CB, Liu CL, Gentles AJ, Chaudhuri AA, Scherer F, et al. Determining cell type abundance and expression from bulk tissues with digital cytometry. Nat Biotechnol. 2019; 37: 773-82.

50. Wu F, Li GZ, Liu HJ, Zhao Z, Chai RC, Liu YQ, et al. Molecular subtyping reveals immune alterations in IDH wild-type lower-grade diffuse glioma. J Pathol. 2020; 251: 272-83.
51. Thorsson V, Gibbs DL, Brown SD, Wolf D, Bortone DS, Ou Yang TH, et al. The Immune Landscape of Cancer. Immunity. 2018; 48: 812-30.e14

52. Bonneville R, Krook MA, Kautto EA, Miya J, Wing MR, Chen HZ, et al. Landscape of Microsatellite Instability Across 39 Cancer Types. ICO Precis Oncol. 2017; 2017.

53. Reinhold WC, Erliandri I, Liu H, Zoppoli G, Pommier Y, Larionov V. Identification of a predominant co-regulation among kinetochore genes, prospective regulatory elements, and association with genomic instability. PLoS One. 2011; 6: e25991.

54. Clijsters L, Hoencamp C, Calis JJA, Marzio A, Handgraaf SM, Cuitino MC, et al. Cyclin F Controls Cell-Cycle Transcriptional Outputs by Directing the Degradation of the Three Activator E2Fs. Mol Cell. 2019; 74: 1264-77.

55. Chen Q, Wang L, Jiang M, Huang J, Jiang Z, Feng H, et al. E2F1 interactive with BRCA1 pathway induces HCC two different small molecule metabolism or cell cycle regulation via mitochondrion or CD4+T to cytosol. J Cell Physiol. 2018; 233: 1213-21.

56. Ma T, Liang F, Oesterreich S, Tseng GC. A Joint Bayesian Model for Integrating Microarray and RNA Sequencing Transcriptomic Data. J Comput Biol. 2017; 24: 647-62.

57. Sung WK, Zheng H, Li S, Chen R, Liu X, Li Y, et al. Genome-wide survey of recurrent HBV integration in hepatocellular carcinoma. Nat Genet. 2012; 44: $765-9$

58. Wurmbach E, Chen YB, Khitrov G, Zhang W, Roayaie S, Schwartz M, et al. Genome-wide molecular profiles of HCV-induced dysplasia and hepatocellular carcinoma. Hepatology. 2007; 45: 938-47.

59. Kim HE, Kim DG, Lee KJ, Son JG, Song MY, Park YM, et al. Frequent amplification of CENPF, GMNN and CDK13 genes in hepatocellular carcinomas. PLoS One. 2012: 7: 43223-35.

60. Dimri M, Satyanarayana A. Molecular Signaling Pathways and Therapeutic Targets in Hepatocellular Carcinoma. Cancers (Basel). 2020; 12: 491-509.

61. Hanahan D, Weinberg RA. Hallmarks of cancer: the next generation. Cell. 2011; 144: 646-74

62. Matsuzaki J, Gnjatic S, Mhawech-Fauceglia P, Beck A, Miller A, Tsuji T, et al. Tumor-infiltrating NY-ESO-1-specific CD8+ T cells are negatively regulated by LAG-3 and PD-1 in human ovarian cancer. Proc Natl Acad Sci U S A. 2010; 107: 7875-80.

63. Wang J, Sanmamed MF, Datar I, Su TT, Ji L, Sun J, et al. Fibrinogen-like Protein 1 Is a Major Immune Inhibitory Ligand of LAG-3. Cell. 2019; 176: 334-47.

64. Hause RJ, Pritchard CC, Shendure J, Salipante SJ. Classification and characterization of microsatellite instability across 18 cancer types. Nat Med. 2016; 22: 1342-50.

65. André T, Shiu KK, Kim TW, Jensen BV, Jensen LH, Punt C, et al. Pembrolizumab in Microsatellite-Instability-High Advanced Colorectal Cancer. N Engl J Med. 2020; 383: 2207-18.

66. Cristescu R, Mogg R, Ayers M, Albright A, Murphy E, Yearley J, et al. Pan-tumor genomic biomarkers for PD-1 checkpoint blockade-based immunotherapy. Science. 2018; 362: 197-206. 